\title{
Searching for Planets Orbiting $\alpha$ Cen A with the James Webb Space Telescope
}

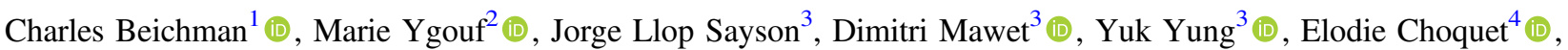 \\ Pierre Kervella ${ }^{5}$ (i), Anthony Boccaletti ${ }^{6}$ (i), Ruslan Belikov ${ }^{7}$, Jack J. Lissauer ${ }^{7}$ (i), Billy Quarles ${ }^{8}$, Pierre-Olivier Lagage ${ }^{9}$, \\ Daniel Dicken ${ }^{9}$, Renyu $\mathrm{Hu}^{10}{ }^{10}$, Bertrand Mennesson $\left.{ }^{10}{ }^{(}\right)$, Mike Ressler ${ }^{10}$, Eugene Serabyn ${ }^{10}$, John Krist $^{10}$, Eduardo Bendek ${ }^{10}$, \\ Jarron Leisenring ${ }^{11}$ (1) and Laurent Pueyo ${ }^{12}$ \\ ${ }^{1}$ NASA Exoplanet Science Institute, Infrared Processing and Analysis Center, Jet Propulsion Laboratory, California Institute of Technology, Pasadena, CA 91125, \\ USA; chas@ipac.caltech.edu \\ ${ }^{2}$ NASA Exoplanet Science Institute, IPAC, Pasadena, CA 91125, USA \\ ${ }^{3}$ California Institute of Technology, Pasadena, CA 91125, USA \\ ${ }^{4}$ Aix Marseille Univ, CNRS, CNES, LAM, Marseille, France \\ ${ }^{5}$ LESIA, Observatoire de Paris, Université PSL, CNRS, Sorbonne Université, Univ. Paris Diderot, Sorbonne Paris Cité, Paris, France \\ ${ }^{6}$ LESIA, Observatoire de Paris, Université PSL, CNRS, Sorbonne Université, Université de Paris, 5 place Jules Janssen, F-92195 Meudon, France \\ ${ }^{7}$ NASA Ames Research Center, Space Science and Astrobiology Division, MS 245-3, Moffett Field, CA 94035, USA \\ ${ }^{8}$ Center for Relativistic Astrophysics, School of Physics, Georgia Institute of Technology, Atlanta, GA 30332, USA \\ ${ }^{9}$ AIM, CEA, CNRS, Université Paris-Saclay, Université Paris Diderot, Sorbonne Paris Cité, F-91191 Gif-sur-Yvette, France \\ ${ }^{10}$ Jet Propulsion Laboratory, California Institute of Technology, Pasadena, CA 91125, USA \\ ${ }^{11}$ Steward Observatory, University of Arizona, Tucson, AZ, USA \\ ${ }^{12}$ Space Telescope Science Institute, Baltimore, MD, USA \\ Received 2019 August 25; accepted 2019 October 14; published 2019 December 12
}

\begin{abstract}
$\alpha$ Centauri A is the closest solar-type star to the Sun and offers an excellent opportunity to detect the thermal emission of a mature planet heated by its host star. The MIRI coronagraph on the James Webb Space Telescope can search the 1-3 au $\left(1^{\prime \prime}-2^{\prime \prime}\right)$ region around $\alpha$ Cen A which is predicted to be stable within the $\alpha$ Cen AB system. We demonstrate that with reasonable performance of the telescope and instrument, a $20 \mathrm{hr}$ program combining on-target and reference star observations at $15.5 \mu \mathrm{m}$ could detect thermal emission from planets as small as $\sim 5 R_{\oplus}$. Multiple visits every 3-6 months would increase the geometrical completeness, provide astrometric confirmation of detected sources, and push the radius limit down to $\sim 3 R_{\oplus}$. An exozodiacal cloud only a few times brighter than our own should also be detectable, although a sufficiently bright cloud might obscure any planet present in the system. While current precision radial velocity (PRV) observations set a limit of 50-100 $M_{\oplus}$ at 1-3 au for planets orbiting $\alpha$ Cen A, there is a broad range of exoplanet radii up to $10 R_{\oplus}$ consistent with these mass limits. A carefully planned observing sequence along with state-of-the-art postprocessing analysis could reject the light from $\alpha$ Cen A at the level of $\sim 10^{-5}$ at $1^{\prime \prime}-2^{\prime \prime}$ and minimize the influence of $\alpha$ Cen B located 7"-8" away in the 2022-2023 timeframe. These space-based observations would complement on-going imaging experiments at shorter wavelengths as well as PRV and astrometric experiments to detect planets dynamically. Planetary demographics suggest that the likelihood of directly imaging a planet whose mass and orbit are consistent with present PRV limits is small, $\sim 5 \%$, and possibly lower if the presence of a binary companion further reduces occurrence rates. However, at a distance of just $1.34 \mathrm{pc}, \alpha$ Cen A is our closest sibling star and certainly merits close scrutiny.
\end{abstract}

Key words: infrared: planetary systems - planetary systems - planets and satellites: detection - space vehicles: instruments

Online material: color figures

\section{Introduction}

The detection, characterization, and search for biomarkers in the atmospheres of Earth analogs in the Habitable Zones (HZs) of their host stars are exciting goals of both ground- and spacebased astronomy as described and prioritized in the National Academy's Decadal Reviews (National Research Council 2010), NASA's Strategic Plan, ${ }^{13}$ the Exoplanet Science Strategy

\footnotetext{
${ }^{13}$ https://www.nasa.gov/sites/default/files/atoms/files/nasa_2018_ strategic_plan.pdf
}

Report $^{14}$ (National Academies of Sciences 2018), and the recently announced Breakthrough Initiative. ${ }^{15}$ The high degree of stellar rejection $\left(10^{-10}\right.$ in the visible and $10^{-7}$ in the thermal infrared) demanded to detect an Earth analog at small angular separations, typically 10 s of milliarcsec for most nearby solar type stars, represents a daunting challenge in both reflected visible light and emitted thermal radiation. Studies of observatories capable of

\footnotetext{
14 https://www.nap.edu/catalog/25187/exoplanet-science-strategy

15 https://breakthroughinitiatives.org/arewealone
} 


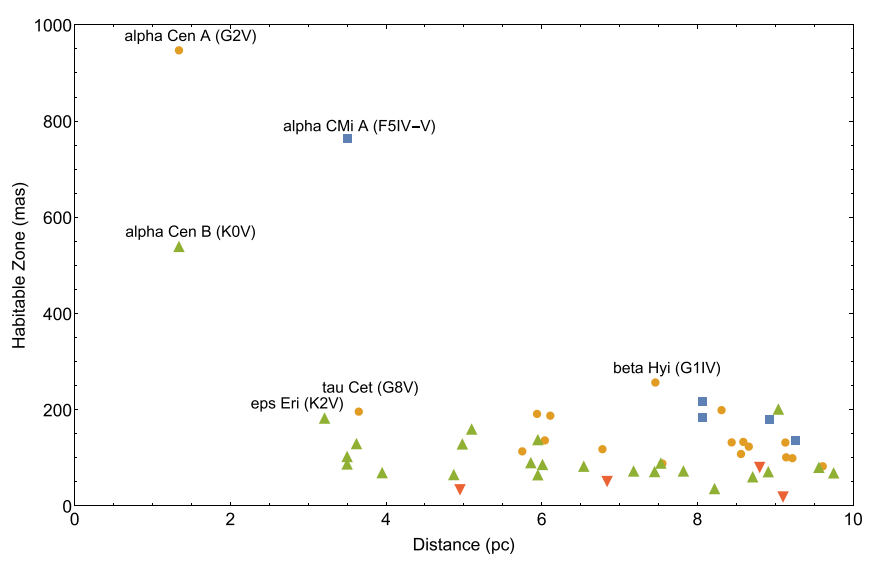

Figure 1. $\alpha$ Cen A stands out as the most favorable star to examine due to the large angular extent of its Habitable Zone, as indicated here as the angular separation (milliarcseconds, or mas) of a planet receiving an Earth equivalent insolation from its host star (Turnbull 2015). A few of the closest and most prominent host stars are called out individually (F stars as blue squares, $G$ stars as orange circles, $\mathrm{K}$ stars as green triangles, and $\mathrm{M}$ stars as inverted red triangles).

(A color version of this figure is available in the online journal.)

achieving these levels have led to designs of instruments for 30-40 m telescopes on the ground (Kenworthy et al. 2016; Mawet et al. 2016; Skemer et al. 2018; Mazin et al. 2019), 4-15 m telescopes in space (Habex, Mennesson et al. 2016; LUVOIR, Pueyo et al. 2017), as well as earlier initiatives such as the TPF-C coronagraph and TPF-I/Darwin mid-IR interferometer (Léger et al. 1996; Angel \& Woolf 1997; Beichman et al. 2007). However, by virtue of its proximity to the Sun, $\alpha$ Cen A offers an opportunity to use more modest and more near-term facilities to image directly a mature planet ranging in size from Jovian-sized to Earth-sized. Proposals exist to use ground-based $8 \mathrm{~m}$ telescopes (Kasper et al. 2017) or a small visible telescope in space (Belikov et al. 2015).

At a distance of $1.34 \mathrm{pc}, \alpha$ Cen $\mathrm{A}$ is 2.7 times closer than the next most favorable $\mathrm{G}$ star, $\tau$ Ceti. $\alpha$ Cen A's luminosity of $1.5 L_{\odot}$ (Thévenin et al. 2002; Kervella et al. 2017) puts the center of its HZ (defined here as the separation of an EarthEquivalent level of insolation, see also Kopparapu et al. 2017) at a physical separation of 1.2 au which corresponds to an angular separation of $0.9 . \alpha$ Cen $\mathrm{A}$ is the one stand-out exception, primum ex parte, in the list of solar-type host stars suitable for the eventual detection and initial characterization of a HZ Earth (Figure 1; Turnbull 2015).

The 10-15 $\mu \mathrm{m}$ emission from an isolated object with the same brightness as a warm Earth-sized planet $(20-40 \mu \mathrm{Jy})$ would be readily detectable by MIRI. There are, of course, major challenges to be overcome: the glare of $\alpha$ Cen A, the presence of $\alpha$ Cen B which might remove planets from the $\alpha$ Cen A system and which introduces a second source of noise, the stability of James Webb Space Telescope (JWST) and the

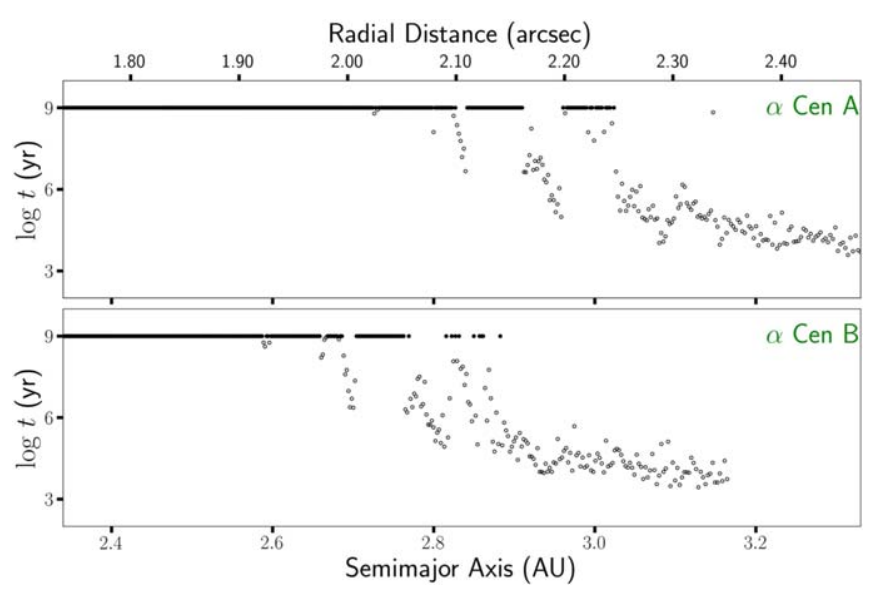

Figure 2. Stable regions are found within $\lesssim 3$ au for planetary systems orbiting $\alpha$ Cen A and within $\sim 2.65$ au of $\alpha$ Cen B (based on work from Quarles et al. 2018).

(A color version of this figure is available in the online journal.)

performance of its coronagraphs. Yet these challenges can be surmounted-certainly for planets larger than the Earth. We note that a search for planets orbiting $\alpha$ Cen $\mathrm{B}$ is less favorable due to the tight radial velocity (RV) constraint on the presence of planets around $\alpha$ Cen B (Zhao et al. 2017), its lower luminosity and correspondingly smaller $\mathrm{HZ}(\sim 0$ !" 5$)$, and to the greater deleterious effects of $\alpha$ Cen A.

Current precision RV (PRV) observations (Zhao et al. 2017) constrain the mass of any planet near $\alpha$ Cen A to be $M \sin (i)<$ $53 M_{\oplus}$ in the HZ (1.2 au). Examination of their Figure 6 which includes their estimates for the effects of non-Gaussian noise sources ("red noise") suggests a limit between 50 and $100 M_{\oplus}$ $(2 \sigma)$. This limit applies to the near edge-on, $79^{\circ}$, orientation of the $\alpha$ Cen A-B system where dynamical studies indicate the presence of a stable zone $\lesssim 3$ au (or 2!"1) around $\alpha$ Cen A despite the presence of $\alpha$ Cen B (Figure 2; Holman \& Wiegert 1999; Quarles \& Lissauer 2016, 2018; Quarles et al. 2018). There is a wide range of planet types possible within these mass limits, from Earth-sized planets to sub-Neptunes. In what follows we adopt an upper limit to any RV signature of $5 \mathrm{~m} \mathrm{~s}^{-1}$ which corresponds roughly to a $2 \sigma$ limit. We recognize that future PRV observations will doubtless improve on this constraint. Finally, we note that a planet's thermal emission depends on its radius, not its mass, and the range of permissible radii is broad due to wide range of observed planet densities.

In this paper we investigate how a modest observing program with the MIRI coronagraph could detect HZ planets larger than $\sim 5 R_{\oplus}$ orbiting $\alpha$ Cen A as well as a zodiacal dust cloud only a few times brighter than our own cloud. Depending on the performance of JWST and the MIRI coronagraph, a more ambitious program could push to even lower planet sizes, $\sim 3 R_{\oplus}$. 


\section{The Prospects for Planets in the $\alpha$ Cen System}

Statistical studies based on RV and transit surveys help to assess whether $\alpha$ Cen A might host one or more planets. Unfortunately, transit surveys are incomplete in the 1-3 au range for all radii (Thompson et al. 2018) while RV data are incomplete for masses below $100 M_{\oplus}$ (Saturn) at these separations (Cumming et al. 2008; Santerne et al. 2016). Combining various estimates suggests a cumulative planet incidence for FGK stars of 3\%-8\% for $M>100 M_{\oplus}$ and $P<5 \mathrm{yr}$ with a five to tenfold increase for masses down to $10 M_{\oplus}$. Thus, based on these statistical considerations there is a good chance (25\%-50\%) that $\alpha$ Cen A might host one or more planets in the $10-100 M_{\oplus}$ range.

Fernandes et al. (2019) parameterize the joint planet occurrence rate as a function of period and mass:

$$
\begin{gathered}
\frac{d^{2} N}{d \log P d \log M}=C_{0} f(P)\left(\frac{M}{10 M_{\oplus}}\right)^{\gamma} \\
f(p)=\left(\frac{P}{P_{\text {break }}}\right)^{p 1} \text { for } P<P_{\text {break }} ; \\
\text { or }\left(\frac{P}{P_{\text {break }}}\right)^{p 2} \text { for } P \geqslant P_{\text {break }} .
\end{gathered}
$$

While there is considerable uncertainty in the fitted parameters, Fernandes et al. (2019) find that the following values provide a reasonable fit to the available data: $P_{\text {break }}=1581$ days, $p 1=$ $-p 2=0.65, \gamma=-0.45$ and $C_{0}=0.84$. If we integrate Equation (1) over periods from 10 to 1800 days (corresponding to an outer limit of $3 \mathrm{au}$ ) with a minimum mass of $10 R_{\oplus}$ and an upper mass consistent with an RV limit of $5 \mathrm{~m} \mathrm{~s}^{-1}$, then $\alpha$ Cen A has a $\sim 15 \%$ probability of hosting a planet with those properties. While the extrapolation to the lowest masses $\left(\sim 10 M_{\oplus}\right)$ is quite uncertain, the population estimates in the mass/radius range which we will show are accessible to JWST $\left(3 \sim 5 R_{\oplus}\right.$ and $P<1800$ days, Section 8) are reasonably well-grounded in transit and RV data (Cumming et al. 2008).

One reason for pessimism about $\alpha$ Cen A's suitability as a stellar parent comes from the fact that $\alpha$ Cen A and B form a relatively tight binary system. Kraus et al. (2016) have analyzed the statistics from Kepler transits and shown that detected planets are only about one-third as abundant in comparable-mass binary systems with projected separations of $<50$ au as they are around single stars. However, Quintana et al. (2002) have shown that the late stages of planet growth for a prograde disk of planetary embryos and planetesimals orbiting about either $\alpha$ Cen A or B near the plane of the binary orbit would grow into a configuration of terrestrial planets comparable to that formed by an analogous disk orbiting the Sun perturbed by Jupiter and Saturn. Xie et al. (2010) and Zhang et al. (2018) have found favorable conditions for planetesimals to survive and grow to planetary embryos in disks with inclinations of up to $10^{\circ}$ relative to the binary orbit. Simulations of Quarles \& Lissauer (2016) and references therein have shown that a planet can remain in a lowinclination, low-eccentricity prograde orbit for longer than the age of the system throughout the HZs of both $\alpha$ Cen A and B.

The population studies mentioned above are given in terms of planet masses, whereas JWST will detect thermal emission which depends on planet radius. For masses between 10 and $100 M_{\oplus}$, radii can range from 2 to $10 R_{\oplus}$ (Howard 2013) with dramatic effects on the photometric signal. We will address the sample consistent with known occurrence rate, the RV limits and detectability by JWST in a subsequent section (Section 8).

\section{Brightness of HZ Planets}

There is a broad base of literature available to establish the expected level of brightness of exoplanets of various sizes and locations (Burrows et al. 2004; Seager \& Deming 2010; Burrows 2014). We have developed a self-consistent series of models based on the atmospheric chemistry and radiative transfer formalism developed in Hu \& Seager $(2013,2014)$. Table 1 and Figure 3 summarize models for 2, 4 and $10 R_{\oplus}$ planets (mini-Neptunes, Neptunes, and Saturns) at 1.2 au with a $\mathrm{H}_{2}$-dominated atmosphere with $10 \times$ solar metallicity. The models include condensation of water, ammonia, and methane when they reach saturation in the atmosphere and are thus suitable to simulate such low-temperature atmospheres. As water condenses in the atmosphere water clouds form at a pressure of $\sim 0.1$ bar. Due to the water clouds, the mid-infrared emission spectrum is close to a $220 \mathrm{~K}$ blackbody as determined by the cloud-top temperature.

For planets at 1.0 au which receive about 50\% more irradiation than Earth our model predicts that water does not condense in its atmosphere and would likely be free of condensation clouds. The spectrum is dominated by strong $\mathrm{H}_{2} \mathrm{O}, \mathrm{CH}_{4}$, and $\mathrm{NH}_{3}$ absorption, as well as $\mathrm{H}_{2}-\mathrm{H}_{2}$ and $\mathrm{H}_{2}-\mathrm{He}$ collision-induced absorption. There are infrared windows into the hot, convective part of the atmosphere, at 4-5 $\mu \mathrm{m}$, and to a lesser extent at $10 \mu \mathrm{m}$.

While it is unlikely that the MIRI observations discussed here will achieve the sensitivity needed to detect Earths or Super-Earths, $\left(1-2 R_{\oplus}\right)$, very long observations combined with new techniques of speckle suppression may allow the detection of rocky planets. Thus, for completeness, we consider some scenarios for small planets (Figure 3(b)), for example a $2 R_{\oplus}$ "water world" for which water is the dominant gas in the atmosphere. A thick water cloud forms with the cloud base at 0.1 bar, and the top at $\sim 0.001$ bar. Due to this thick cloud that extends to low pressures, the resulting spectrum is a blackbody at $215 \mathrm{~K}$. We also considered an Earth-like planet, with either 1 or 1.4 $R_{\oplus}$ (an Earth or Super-Earth). We simulated the atmosphere using the standard, mid-latitude temperature-pressure profile and 
Table 1

Predicted Brightness of Possible Planets Orbiting $\alpha$ Cen A

\begin{tabular}{|c|c|c|c|c|c|c|}
\hline $\begin{array}{l}\text { Planet } \\
\text { Type }\end{array}$ & $\begin{array}{c}\text { Radius } \\
\left(R_{\oplus}\right)\end{array}$ & $\begin{array}{l}\text { Orbit } \\
\text { (au) }\end{array}$ & $\begin{array}{l}T_{\text {eff }} \\
(\mathrm{K})\end{array}$ & $\begin{array}{c}\mathrm{F} 1065 \mathrm{C} \\
(\mu \mathrm{Jy})\end{array}$ & $\begin{array}{c}\mathrm{F} 1550 \mathrm{C} \\
(\mu \mathrm{Jy})\end{array}$ & $\begin{array}{c}F_{\mathrm{pl}} / F_{*} \\
\mathrm{~F} 1550 \mathrm{C}^{\mathrm{a}}\end{array}$ \\
\hline Saturn & 10 & 1.2 & 221 & 500 & 1210 & $1.9 \times 10^{-5}$ \\
\hline Warm Saturn & 10 & 1.0 & 275 & 1370 & 2380 & $3.7 \times 10^{-5}$ \\
\hline Neptune & 4 & 1.2 & 221 & 80 & 190 & $0.3 \times 10^{-5}$ \\
\hline Warm Neptune & 4 & 1.0 & 262 & 220 & 380 & $0.6 \times 10^{-5}$ \\
\hline Mini-Neptune & 2 & 1.2 & 221 & 20 & 50 & $0.08 \times 10^{-5}$ \\
\hline Warm Mini-Neptune & 2 & 1.0 & 262 & 55 & 95 & $0.15 \times 10^{-5}$ \\
\hline Earth & 1.0 & 1.2 & 250 & 40 & 20 & $0.03 \times 10^{-5}$ \\
\hline ExoZodi Cloud ${ }^{\mathrm{b}}$ & $\ldots$ & 0.75 to 1.77 & $250-300$ & 2500 & 3000 & $3.6 \times 10^{-5}$ \\
\hline
\end{tabular}

Notes.

${ }^{\text {a }}$ Contrast $F$ (planet) $/ F$ (star).

${ }^{\mathrm{b}}$ Estimated brightness of an analog of the solar system's zodiacal cloud as discussed in Section 4.
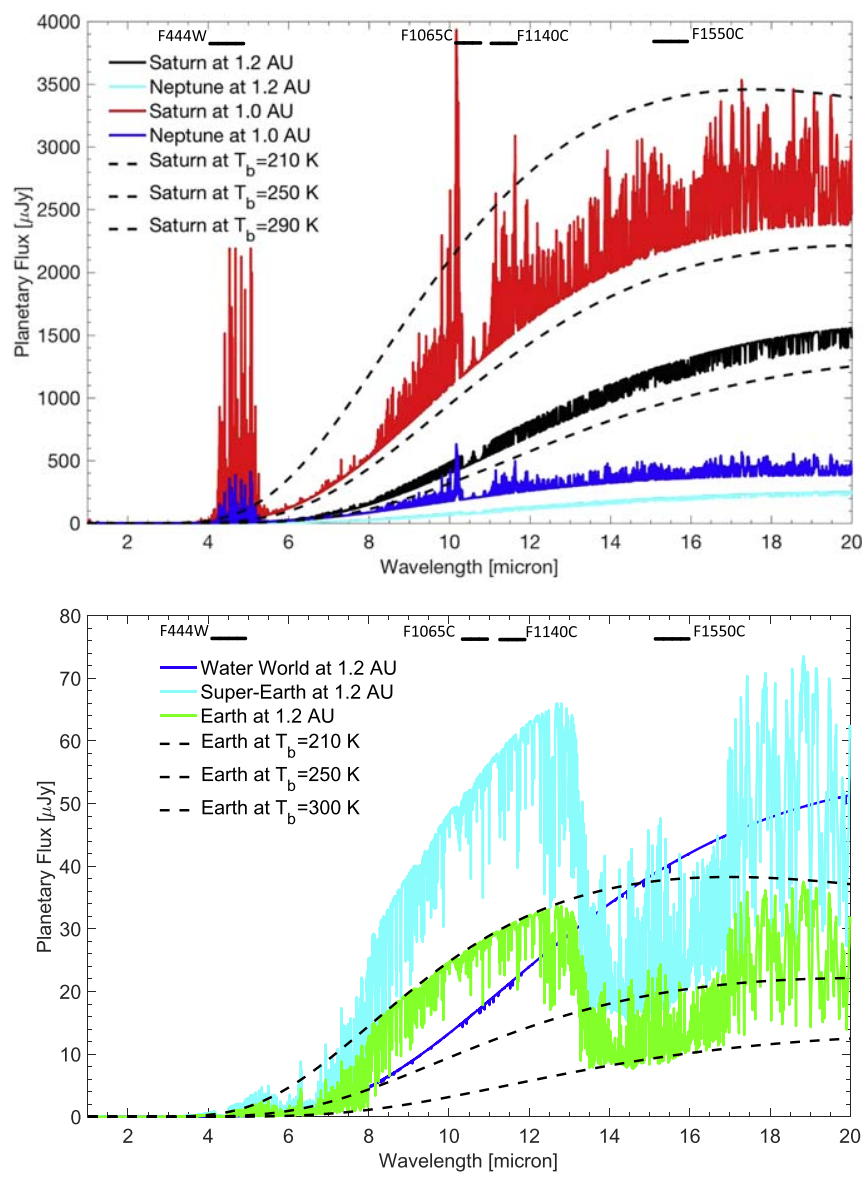

Figure 3. The brightness of a variety of model planets with radii between 4 and $10 R_{\oplus}$ ((a), top) and 1-2 $R_{\oplus}$ ((b), bottom) over a range of orbital locations and temperatures as described in Table 1. The locations of the 3 MIRI coronagraphic filters and one NIRCam filter are indicated.

(A color version of this figure is available in the online journal.) the full photochemistry model developed in $\mathrm{Hu}$ et al. (2012). It is well known that thermal emission of Earth can be presented by a combination of cloud-free, low-altitude cloud, and high-altitude cloud atmospheres, e.g., (Des Marais et al. 2002; Turnbull et al. 2006). But for simplicity, we assumed a cloud-free atmosphere noting that other cloud types have smaller thermal emission features. The emission spectrum is dominated by absorption of $\mathrm{CO}_{2}, \mathrm{H}_{2} \mathrm{O}$, and $\mathrm{O}_{3}$.

Finally, for subsequent analyses (Sections 6.2,8), we also used a simple blackbody relationship (Traub \& Oppenheimer 2010):

$$
\begin{aligned}
T_{\text {eff }} & =T_{*}\left(\frac{1-A}{4 f}\right)^{1 / 4}\left(\frac{R_{*}}{d}\right)^{1 / 2} \\
& =275 L_{*}^{1 / 4}(1-A)^{1 / 4} d^{-1 / 2} \mathrm{~K}
\end{aligned}
$$

where $L_{*}, T_{*}, R_{*}$ are the stellar luminosity, effective temperature and radius, $A$ the planet albedo, $d$ the planet's distance from the star in astronomical unit (Figure 4), and $f=1$ is appropriate for full heat distribution. In the figure, the adopted albedo is 0.3 , but in subsequent analyses, the albedo was drawn randomly between 0.15 and 0.65 appropriate to gaseous planets in our solar system, e.g., Cahoy et al. (2010).

\section{Exozodiacal Dust Orbiting $\alpha$ Cen A}

The zodiacal cloud and Kuiper Belt in our solar system have analogs in many other planetary systems. The recently published HOSTS survey used the nulling interferometer of the Large Binocular Telescope to set preliminary upper limits of 26 times the solar system zodiacal level for a sample of solar type stars (Ertel et al. 2018). Wiegert et al. (2014) find suggestive, but hardly definitive evidence for a ring of cold dust (53 K) 


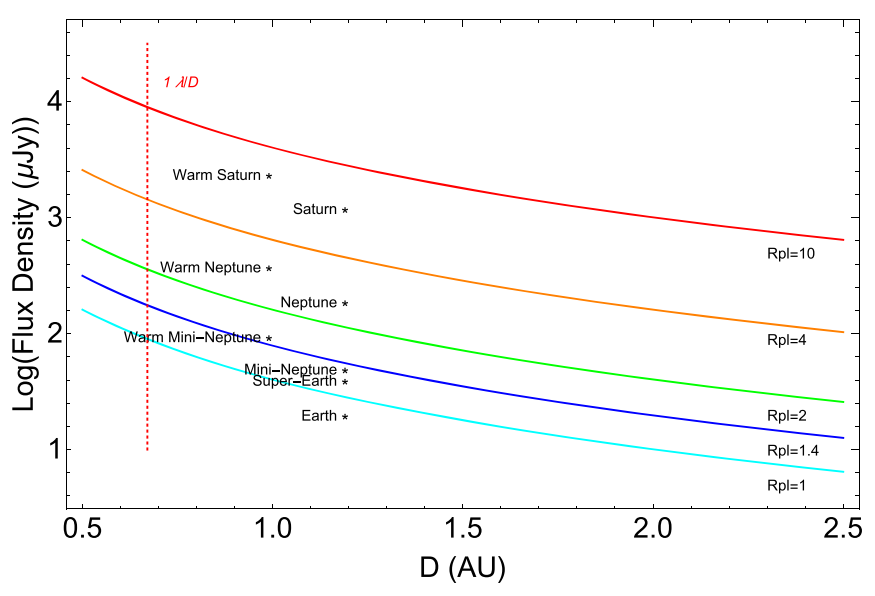

Figure 4. The lines show the flux density at F1550C for planets of different radii (denoted in $R_{\oplus}$ on the right) as a function of radial separation from $\alpha$ Cen A based on a simple $T_{\text {eff }} \propto D^{0.5}$ relationship for an albedo of 0.3. Also shown are the predicted F1550C flux densities for the detailed models specified in Table 1. The dotted red vertical line shows the projected location of MIRI's $1 \lambda / D=0$ ". 67 Inner Working Angle at $15.5 \mu \mathrm{m}$.

(A color version of this figure is available in the online journal.)

located at $\sim 70-105$ au around the $\alpha$ Cen AB system at a level comparable to the Edgeworth-Kuiper Belt in our own solar system (Teplitz et al. 1999). The HOSTS survey suggests that the level of warm zodiacal emission is higher for stars associated with cold dust emission detected at longer wavelengths by Spitzer or Herschel.

The proximity of $\alpha$ Cen means that JWST/MIRI can spatially resolve a warm zodiacal dust cloud without an interferometer and thereby improve the detectability of the dust relative to purely photometric measurements (Beichman et al. 2006b). A model of a near-edge-on " 1 zodi" cloud seen around $\alpha$ Cen A at $15.5 \mu \mathrm{m}$ can be generated using ZodiPic (Kuchner 2012). Figure 5 shows an $5^{\prime \prime} \times 5^{\prime \prime}$ image of a cloud whose total dust flux density is $8.9 \mathrm{mJy}$, i.e., about $10^{-4}$ of the stellar flux at the same wavelength. Adopting an "optimistic" HZ definition of 0.75-1.77 au (Kopparapu et al. 2017) for a Sun-like star and correcting for stellar luminosity, the excess flux density in the $\mathrm{HZ}$ is approximately $3 \mathrm{mJy}$, i.e., a total fractional excess of $3.6 \times 10^{-5}$ between 0.92 and 2.18 au. This emission would be spread over roughly 10 MIRI beams, or approximately $0.3 \mathrm{mJy}$ per beam which is comparable in brightness to a "Warm Neptune" (Table 1). The detection of emission at this level (Section 7) is interesting for two reasons. First, observing a spatially resolved excess in the $\mathrm{HZ}$ would be an important contribution to our knowledge of the evolution of exoplanet systems. Second, exozodiacal emission at the few Zodi level may set a limit to the size of a $\mathrm{HZ}$ planet which might be detectable with MIRI's angular resolution (Beichman et al. 2006a).

Exactly how the exozodiacal dust is distributed is critical to its detectability and its effect on the detectability of any planets. Many

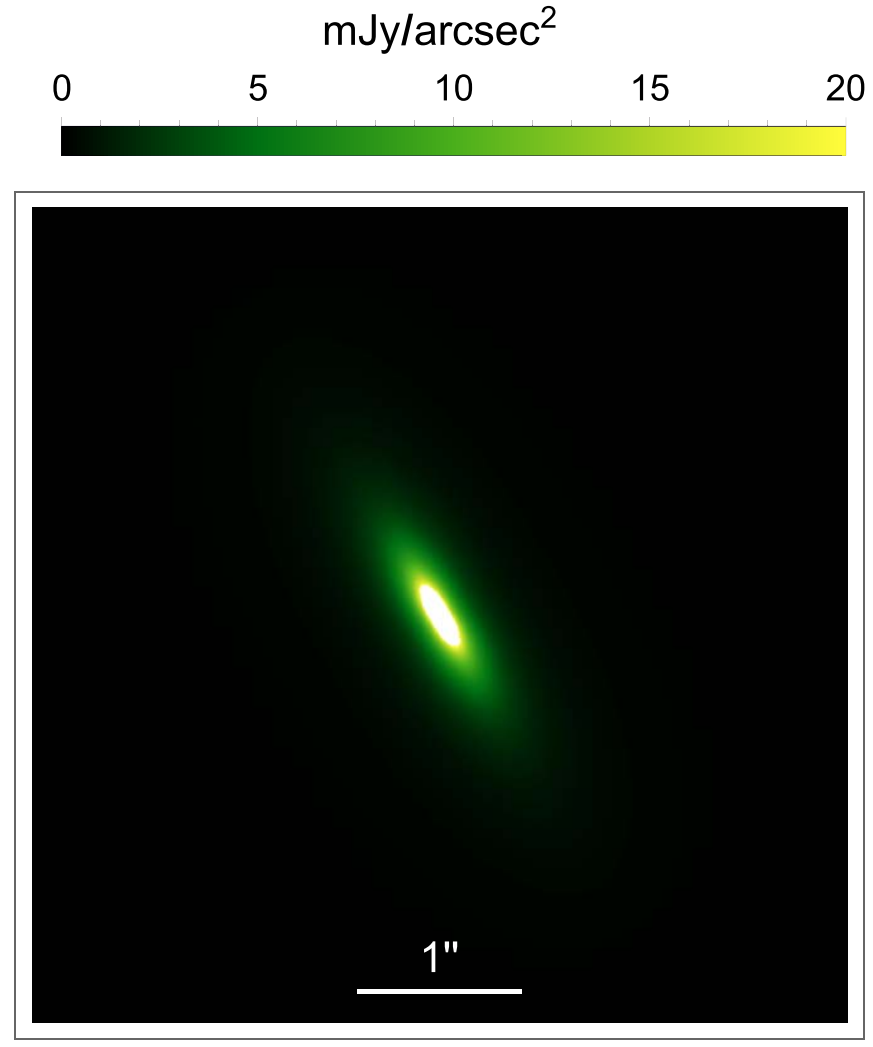

Figure 5. A model of a " 1 zodi" cloud seen around $\alpha$ Cen A at $15.5 \mu \mathrm{m}$, generated using ZodiPic (Kuchner 2012) for a disk seen nearly edge-on $\left(79^{\circ}\right)$. The image is $5^{\prime \prime}$ on a side. The total dust flux is $8.9 \mathrm{mJy}$, i.e about $10^{-4}$ of the stellar flux at the same wavelength.

(A color version of this figure is available in the online journal.)

exozodiacal clouds, e.g., Fomalhaut, HD69830, $\epsilon$ Eridani, have gaps rings or clumps often attributed to the presence of planets (Beichman et al. 2005; Su et al. 2013; Mawet et al. 2019). A faint but homogeneous disk might simply be resolved away during the reference star subtraction while a clumpy cloud observed at the limit of JWST's angular resolution might be confused with one or more planets. Additional simulations and finally JWST observations will be required to assess these challenges.

\section{Overcoming the Observational Challenges}

The first challenge to finding one or more planets orbiting $\alpha$ Cen $\mathrm{A}$ is to select the preferred wavelength and instrument. Compared to NIRCam's coronagraph operating at 4-5 $\mu \mathrm{m}$ with an Inner Working Angle (IWA) of 4-6 $\lambda / D$, MIRI's Four Quadrant Phase Mask (4QPM) operating at $\sim 1 \lambda / D$ offers: comparable IWA, improved immunity to Wavefront Error (WFE) drifts and centering errors (Knight et al. 2012), more favorable planet-star contrast ratio at the expected planet temperatures at the IWA (200-300 K; Figure 3), and lower brightness of background stars. MIRI offers three 4QPM masks 


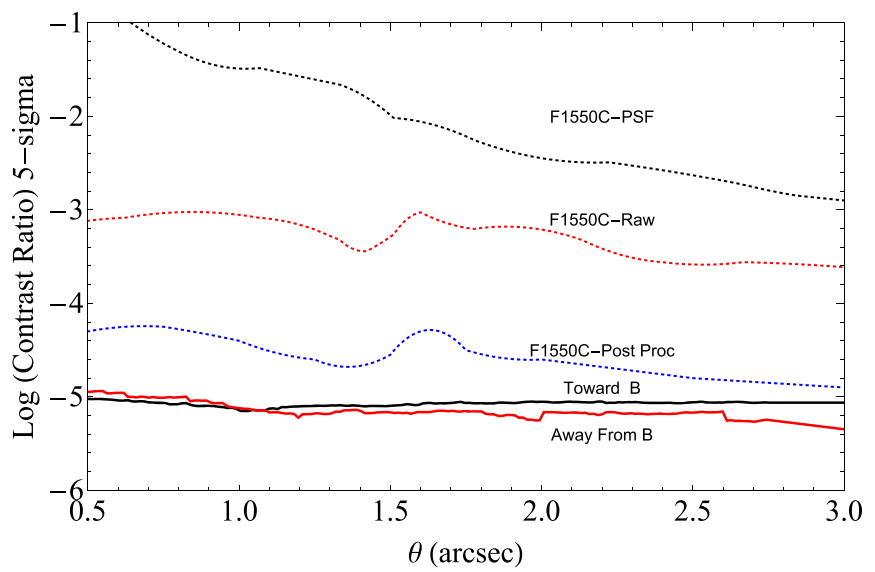

Figure 6. Contrast curves for the F1550C curve: PSF (dotted, black), Raw coronagraphic contrast (dotted, red), post PSF subtraction (dotted, blue)—all from (Boccaletti et al. 2015). The two solid curves show the contrast following our PCA post-processing with the upper black curve showing the influence in the direction of $\alpha \mathrm{Cen} \mathrm{B}$, located 7" away, and the lower red curve the contrast in directions away from $\alpha$ Cen B. The effect of $\alpha$ Cen B is negligible with a few arcseconds of $\alpha$ Cen A.

(A color version of this figure is available in the online journal.)

at $10.65,11.4$ and $15.5 \mu \mathrm{m}$. Although the shortest wavelength filter would have a smaller IWA, we have focused our discussion on $\mathrm{F} 1550 \mathrm{C}$ for a number of reasons: longer integration time before detector saturation ${ }^{16}(10 \mathrm{~s}$ versus $1 \mathrm{~s}$ for F1550C versus F1065C), lower impact of wavefront drifts, good sensitivity across a broad range of planet temperatures (Figure 3), lower confusion due to background stars, and complementarity to shorter-wavelength ground-based efforts (Section 8.2).

\subsection{Rejecting Starlight from $\alpha$ Cen A}

MIRI's 4QPM reduces the central brightness of a star by a factor of $\sim 10^{3}$, operates as close to the star as $1 \lambda / D \sim 0$." 48 at $\lambda=15.5 \mu \mathrm{m}$ (where $D$ is the telescope diameter), and achieves $10^{-4}-10^{-5}$ rejection at a separation of $1^{\prime \prime}-2^{\prime \prime}$ using standard reference star subtraction (Boccaletti et al. 2015, Figure 6). As we discuss in Section 6.2 and show in the two lower lines in Figure 6, it should be possible to improve on this performance with a specialized observing mode and advanced post-processing.

\subsection{Rejecting Starlight from $\alpha$ Cen $B$}

Complicating the issue is the presence of $\alpha \mathrm{Cen} \mathrm{B}$ which will be located roughly $7^{\prime \prime}-8^{\prime \prime}$ away from $\alpha$ Cen B during the first few years after JWST's launch. (Figure 7). We considered two methods for dealing with $\alpha$ Cen B: (1) placing $\alpha$ Cen B on the transmission gap in the 4QPM (Boccaletti et al. 2015;

\footnotetext{
${ }^{16}$ As calculated using the JWST exposure time tool. https://jwst.etc.stsci. edu/.
}

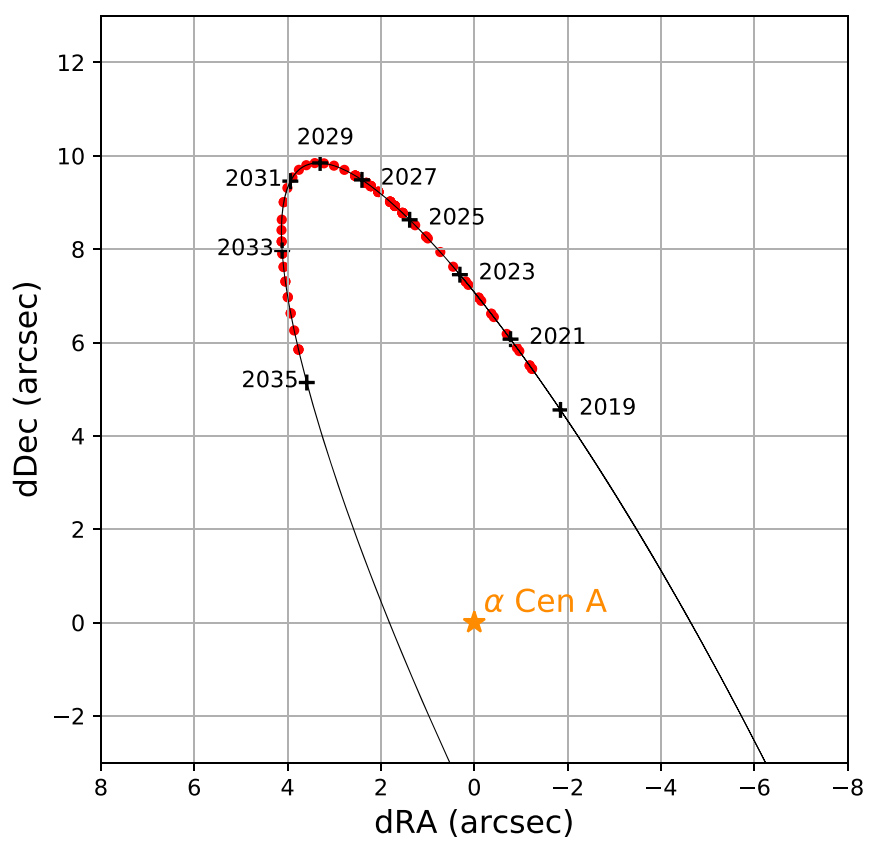

Figure 7. The orbit of $\alpha$ Cen B around $\alpha$ Cen A (Kervella et al. 2016) is indicated with some possible observing dates $(>2021)$ highlighted during the early years of JWST's operation.

(A color version of this figure is available in the online journal.)

Danielski et al. 2018) to reduce the its central intensity at the cost of a limited selection of observing dates with the correct on-sky orientation; or (2) optimize the target-reference star observations so as to minimize wave front error drifts while accepting the deleterious effects of the full brightness of $\alpha$ Cen $\mathrm{B}$ falling on the detector.

A positive aspect of the $4 \mathrm{QPM}$ coronagraphic masks is the existence of a gap, \pm 3 pixels (at the half power points) or $\sim 0$ !' 3 , located at the phase boundaries of the four quadrants. At these locations the transmission is reduced by a factor of $>8$ (Danielski et al. 2018). There are semi-annual observing windows of a few days duration during which $\alpha$ Cen A can be centered behind the coronagraphic mask while at the same time placing $\alpha$ Cen $\mathrm{B}$ in one of the gaps between adjacent quadrants, thereby reducing detector artifacts. However, as discussed in Section 6.2 this approach requires a non-optimized slew to a reference star which may induce changes in telescope's thermal environment resulting in non-zero wavefront errors and a higher level of residual speckles.

The alternative approach of placing $\alpha$ Cen B in an unattenuated portion of the detector offers the advantage of a broader observing window at the cost of a greater risk of deleterious effects of the full intensity of $\alpha$ Cen B.

\subsection{Confusion by Background Stars and Galaxies}

The high proper motion of $\alpha$ Cen $\left(\sim 3^{\prime \prime} \mathrm{yr}^{-1}\right.$ due West) means that images from earlier epochs (Spitzer, HST, ground-based, etc.) 


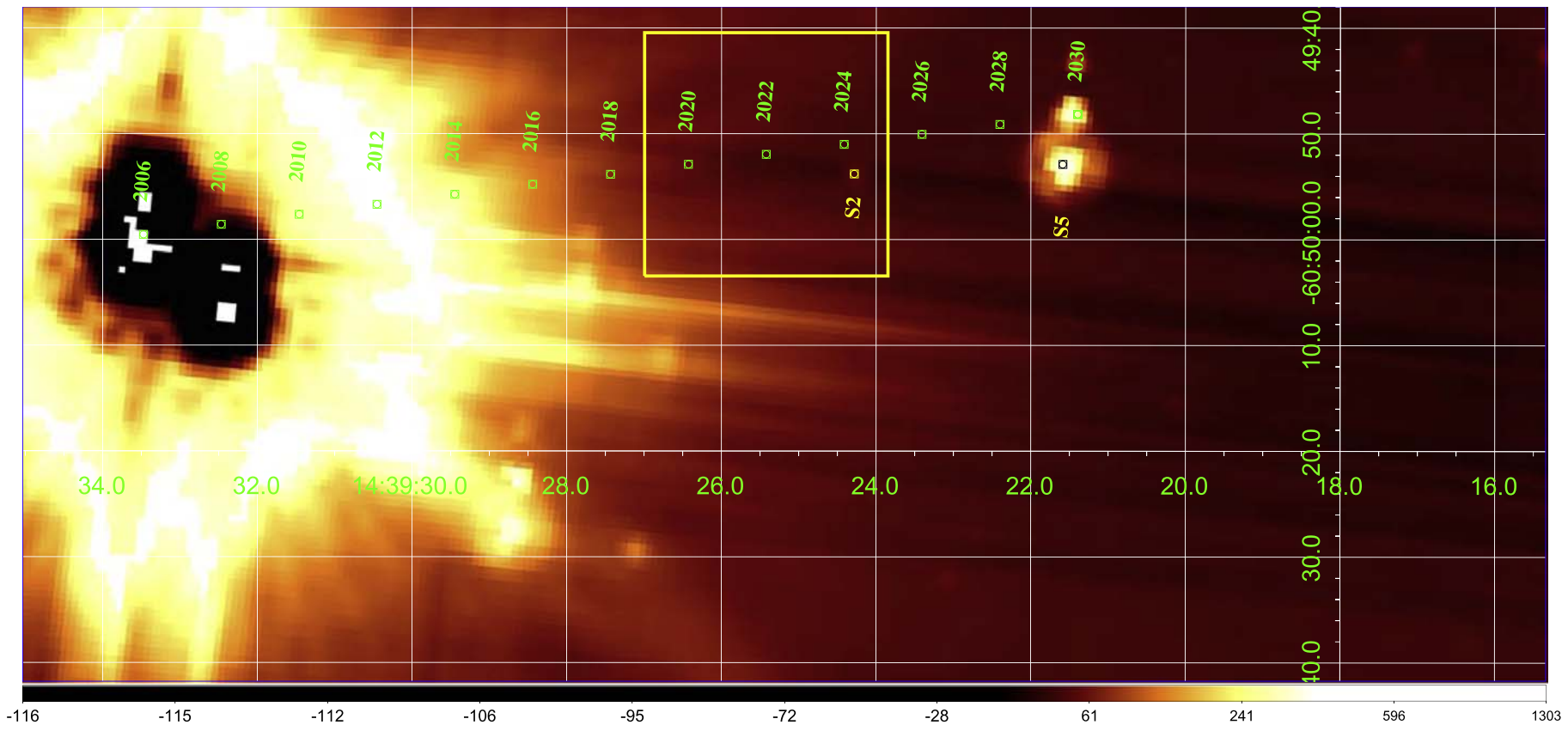

Figure 8. A Spitzer image (in celestial coordinates) of $\alpha$ Cen AB (Fazio \& Megeath 2004) taken in 2005 at $8.0 \mu \mathrm{m}$. The position of $\alpha$ Cen A in 2022 is shown with a yellow square demarcating the approximate field of the $23^{\prime \prime}$ MIRI coronagraph. There are no Spitzer sources within the projected MIRI field at the level of a few mJy. The approximate position of $\alpha$ Cen A is shown by a series of green squares through 2030 when the source labeled "S5" (Kervella et al. 2016) approach $\alpha$ Cen A itself. (A color version of this figure is available in the online journal.)

can be used to study the field where $\alpha$ Cen will be during the $J W S T$ era and to identify background objects. Figure 8 shows a $8 \mu \mathrm{m}$ (Ch 4) Spitzer/IRAC image of $\alpha$ Cen AB taken in 2005 (Fazio \& Megeath 2004) with the location of MIRI's 23" coronagraphic field surrounding $\alpha$ Cen's projected position around $\sim 2022$. The brightest stars in the vicinity are $S 2$ $\left(K_{s}=11.1 \mathrm{mag}\right.$ ) which will pass within 1". 6 of $\alpha$ Cen A around 2023.4 and a brighter source $S 5\left(K_{s}=7.8 \mathrm{mag}\right)$ which will pass within 0"'015 in 2028.4 (Kervella et al. 2016). The impending approach of $S 2$ argues for observing $\alpha$ Cen A soon after launch to avoid the impact of $S 2$ on the observations.

Even if there are no obvious bright stars in the coronagraphic field it is important to estimate the level of contamination of background stars and galaxies at the expected levels of emission for our hoped-for planets, e.g., $F_{\nu}(\mathrm{F} 1550 \mathrm{C})=20 \mu \mathrm{Jy}$ for an Earth analog and $2.4 \mathrm{mJy}$ for a warm Saturn (Table 1). To estimate the stellar background we take advantage of Spitzer's GLIMPSE survey (Churchwell et al. 2009) which covered a region near $\alpha$ Cen. We extracted from the Deep GLIMPSE catalog ${ }^{17}$ sources in a $r=10^{\prime}$ region located at galactic coordinates $(l, b)=\left(-315^{\circ} .3,-0.56\right)$, just 0.5 away from $\alpha$ Cen, at $4.6 \mu \mathrm{m}$. The $4.6 \mu \mathrm{m}$ data become confusion limited ( $\sim 50$ beams per source) at around $500 \mu \mathrm{Jy}$ (Figure 9), but at fluxes brighter than this level the plot of cumulative source counts, $N$, as

\footnotetext{
${ }^{17}$ https://irsa.ipac.caltech.edu/data/SPITZER/GLIMPSE/gator_docs/ GLIMPSE_colDescriptions.html
}

a function of flux density, $S, \log N(>S) / \log S$ has a slope of -0.77 , typical of a distribution of stars in the Galactic plane.

If we extrapolate the source counts to lower fluxes assuming that most of these objects have a Rayleigh-Jeans spectrum, then we can estimate the number of background sources expected within a \pm 2 !"5 (3.3 au) field around $\alpha$ Cen A at F1550C. The extrapolated number of $15.5 \mu \mathrm{m}$ sources is 0.004 at the $2.4 \mathrm{mJy}$ brightness of a Saturn and 0.15 sources for a $20 \mu \mathrm{Jy}$ Earth (Table 1). Only at the brightness level of an Earth does the expected occurrence of background stars become a matter of concern, while for a Neptune the expected number of background sources in a \pm 2 !" 5 field is 0.03 . The expected number of extra-galactic background sources is even lower at these flux levels. Using model sources counts from Cowley et al. (2018) we find that the predicted number of galaxies at $15 \mu \mathrm{m}$ within $2^{\prime \prime}$ of $\alpha$ Cen A is less than 0.0035 at $20 \mu \mathrm{Jy}$. For host stars $10-20$ times further away than $\alpha$ Cen A, the incidence of stellar and especially extra-galactic background objects will be a much more serious problem. Even though the stellar and extra-galactic sources of false positives are rare, multi-color (F1065C versus F1550C) and ultimately astrometric confirmation will be required to confidently reject background objects.

\subsection{Detector Performance Toward Bright Stars}

Stars as bright as $\alpha$ Cen $\mathrm{AB}$ present unique challenges for the MIRI detector which is a $1024 \times 1024$ arsenic-doped 


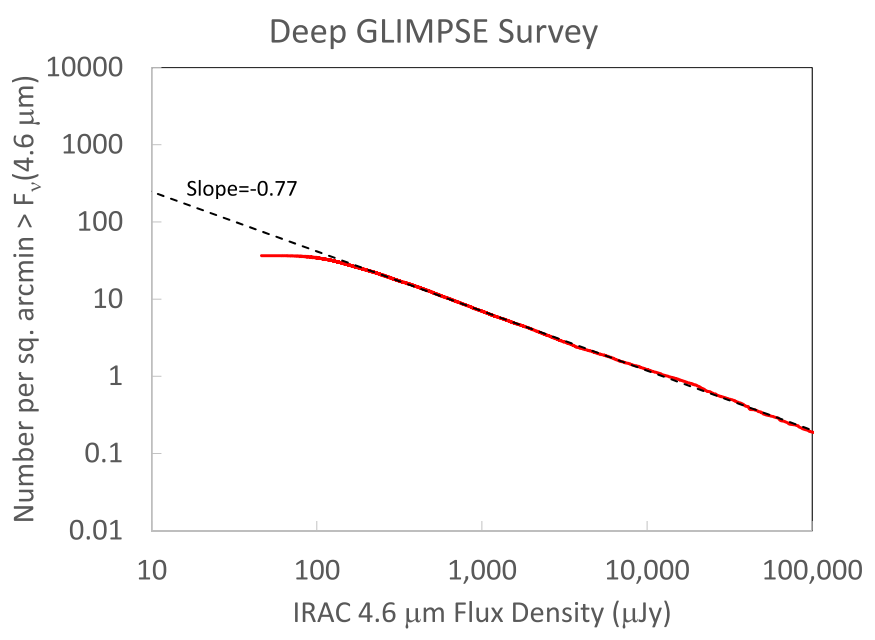

Figure 9. Spitzer star counts at $4.6 \mu \mathrm{m}$ from the GLIMPSE survey at a position close to $\alpha$ Cen are extrapolated below the confusion limit. The slope of the curve is typical of stellar populations in the Galactic Plane. We assume that background stars are fainter at the $15.5 \mu \mathrm{m}$ wavelength of the MIRI coronagraph by a Rayleigh-Jeans factor of $(15.5 \mu \mathrm{m} / 4.6 \mu \mathrm{m})^{2}=11.3$.

(A color version of this figure is available in the online journal.)

silicon (Si:As) IBC hybrid array (Ressler et al. 2015; Rieke et al. 2015). Even if placed behind one of the gaps in the 4QPM mask, $\alpha$ Cen B would saturate portions of the detector and if not attenuated by a gap, the saturation problems would be even worse. To address detector artifacts from very bright sources, we used an instrument testbed at JPL to conduct tests on an MIRI engineering model detector using an exact copy of the flight electronics. Appendix describes the test results in detail, but the primary conclusion is that the tests reveal no detectorbased limitations to the detection of planets around the $\alpha$ Cen A.

\section{Observational Scenarios}

The signal-to-noise ratio $(\mathrm{S} / \mathrm{N})$ of a detection near $\alpha$ Cen $\mathrm{A}$ is driven by both photon noise due to unsuppressed starlight which can be mitigated with increasing integration time and residual speckle noise which must be mitigated via improved point-spread function (PSF) and speckle suppression. The envisioned technique of post-processing relies on the observation of a reference star with the small-grid dither technique. This technique compensates for possible jitter during the observation that slightly change the position of the target behind the coronagraph by artificially reproducing the same jitter effect while observing the reference star.

Our simulations of the observational sequence show that we achieve a reasonable balance between photon noise and residual speckle noise if we set the number integrations per dither point to keep the ratio of total target to reference star observing time at 1:3. This ratio depends on the difference of magnitude between the target and the reference and the stability of the observations. In particular, it is a compromise between two extreme scenarios: (1) negligible level of jitter that would require a 1:9 ratio or (2) higher level of jitter that would allow a ratio closer to $1: 1$, assuming two stars of the same magnitude. The adopted 1:3 ratio is a compromise that we would refine with further simulations and on-orbit information on the performance of JWST.

With this plan we can achieve detections at the levels at the $10^{-5}$ level at $>1^{\prime \prime}$ as discussed below. An initial reconnaissance program sufficient to detect a $5 \sim 6 R_{\oplus}$ planet would require approximately $3.5 \mathrm{hr}$ of on-target observing time. Adding in the $\sim 3 \times$ longer duration of reference star observation plus observatory overheads leads to a total $\sim 20 \mathrm{hr}$ program according to the JWST Exposure Time Calculator. ${ }^{18}$

A single epoch of F1550C observations will produce a data set which will both probe the limits of MIRI coronagraphy and result in either the detection of a planet or set limits at the $5 \sim 6 R_{\oplus}$ level. MIRI might also detect solar system levels of exozodiacal emission (Section 7). Subsequent observations at multiple wavelengths would identify background objects with stellar colors and provide astrometric confirmation of detected objects.

\subsection{Reference Star Selection}

Coronagraphic imaging to detect a $5 R_{\oplus}$ planet, not to mention $1 R_{\oplus}$, presents a daunting observational challenge. The choice of a reference star is critical to removing the stellar PSF and residual speckles. To minimize observing time on the reference star and to maximize the level of speckle suppression it is important to find the best match in terms of brightness, spectral type and angular separation. Fortunately, on the Rayleigh-Jeans tail of photospheric emission, color effects in the narrow $6 \%$ passbands of F1550C filters are small compared with shorter wavelength observations.

There are a number of options for reference star which also affect the overall observing scenario. The closest reference to $\alpha$ Cen A is, of course, $\alpha$ Cen B. Using $\alpha$ Cen B has the advantages of minimal change in telescope configuration and rapid target acquisition compared with choosing a more distant reference star. The disadvantage is that one can never escape the influence of the $\sim 1 \mathrm{mag}$ (at long wavelengths) brighter $\alpha$ Cen A to obtain a clean, uncontaminated PSF measurement. Ground-based programs have adopted the $\alpha$ Cen B approach using rapid chopping between the two stars (Section 8.2). Here we examine a more conservative approach which takes a more widely separated, single star to evaluate the PSF at the positions of both $\alpha$ Cen A and B. Interestingly, the two scenarios require roughly the same amount of wall clock time as determined by the JWST APT tool, ${ }^{19}$ approximately $20 \mathrm{hr}$.

\footnotetext{
18 https://jwst.etc.stsci.edu/

19 http://www.stsci.edu/scientific-community/software/astronomersproposal-tool-apt
} 
Table 2

Candidate Reference Stars

\begin{tabular}{lccc}
\hline \hline Star & Spec Type & Sep $(\mathrm{deg})$ & {$[(12 \mu \mathrm{m})] \mathrm{mag}^{\mathrm{a}}$} \\
\hline BL Cru & M4/5 III & 17 & -0.93 \\
BO Mus & M6II/III & 15 & -1.7 \\
DL Cha & M6III & 18 & -0.64 \\
V996 Cen & Carbon Star & 8 & -0.70 \\
$\epsilon$ Mus & M4III & 17 & -2.09 \\
del01 Aps & M4III LPV & 20 & -1.36 \\
$\zeta$ Ara & K3III & 19 & -1.17 \\
\hline$\alpha$ Cen B & K1V & 0.002 & $-0.6^{\mathrm{b}}$ \\
\hline
\end{tabular}

Notes.

a Magnitude from IRAS Catalog.

${ }^{\mathrm{b}}$ Estimated from shorter wavelengths.

For stars as bright as $[\mathrm{F} 1555 \mathrm{C}] \sim-1.4 \mathrm{mag}$, our choices are quite limited. We used the IRAS Low Resolution Spectrometer Catalog (Olnon et al. 1986) to identify potential reference stars: $F_{\nu}(12 \mu \mathrm{m})>50 \mathrm{Jy}$ within $20^{\circ}$ of $\alpha$ Cen, clean Rayleigh-Jeans photospheric emission, constant ratio $(<10 \%)$ of LRS brightness $(F(\alpha$ Cen $) / F($ star $))$ across the $\mathrm{F} 1550 \mathrm{C}$ band, a low probability of variability during the 300 days IRAS mission (VAR $<15 \%)$, and no bright companions within $100^{\prime \prime}$. Table 2 lists potential reference stars. The ratio of the LRS spectra of the (unresolved) $\alpha$ Cen $\mathrm{AB}$ system to these stars is constant across the F1550C bandpass to $<1 \%$.

\subsection{Achieving Highest Imaging Contrast}

Achieving the sensitivity needed to detect planets requires aggressive post-processing techniques to reduce the residual speckles from both $\alpha$ Cen A and B. We have simulated an observing scenario which places a reference star at the positions of both $\alpha$ Cen A and B. The small grid 9-point dither pattern available for MIRI observations is used at the position of $\alpha$ Cen A. The 15 mas microsteps in the dither pattern combined with the 6.7 mas pointing jitter during the observation ${ }^{20}$ improve the sampling of the PSF and thus the ability to remove stellar speckles (Figure 10). We used a Principal Component Analysis (PCA) Algorithm (Amara \& Quanz 2012; Soummer et al. 2012) to generate a sequence of reference images using all the individual short-exposure frames obtained during the observations.

For each image we generated a wavefront map realization which differed from its predecessor by a random amount and by a linear drift as described by Perrin et al. (2018) and which will be described in more detail below. The resultant wavefront maps were used to create two PSFs using the IDL version of MIRImSIM ${ }^{21}$ : the on-axis PSF representing $\alpha$ Cen $\mathrm{A}$ and an

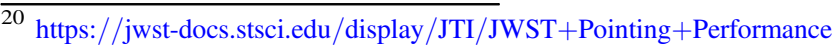

${ }^{21}$ https://jwst.fr/wp/?p=30
}

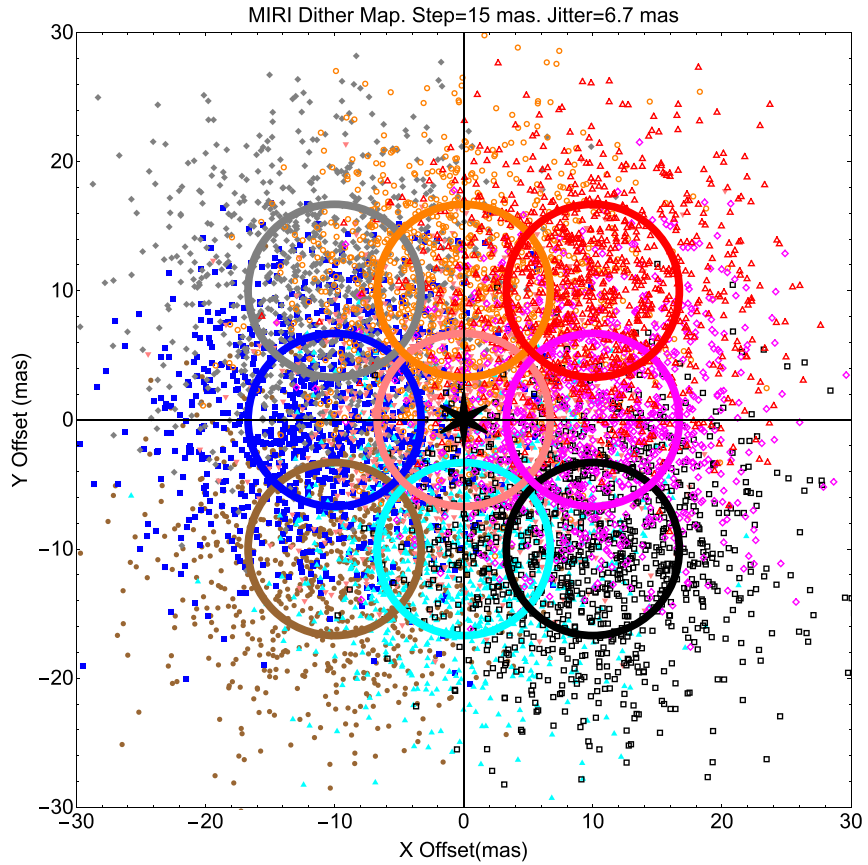

Figure 10. The 9 point grid dither observation strategy combined with the diversity added by the 6.7 mas jitter of the telescope (denoted by the circles) during acquisition, allows for enhanced diversity in the reference images (each denoted by a small symbol) to be used for reduction.

(A color version of this figure is available in the online journal.)

off-axis PSF at 7 " representing $\alpha$ Cen $\mathrm{B}$ at its projected separation in $\sim 2022$. For this simulation we generated 468 exposures (52 separate pointings each with a 9 point dither pattern) for reference star at the position of $\mathrm{A}$ and 100 pointings (with no dither) for the reference star at the position of B. These individual reference star images were combined to generate a PSF library with 25,000 individual images (out of a possible $46,800)$ of the $\alpha$ Cen AB system.

We also generated 200 images of $\alpha$ Cen A including planets of different sizes and locations $\left(1-10 R_{\oplus}, 0\right.$ " $\left.5-3^{\prime \prime}\right)$. We also generated over 450 reference star images (Section 6.2.1). On orbit we will obtain many more images by using short exposures, $\sim 10 \mathrm{~s}$, to avoid saturation at the core of $\alpha$ Cen A and to further increase image diversity. ${ }^{22}$ Experimenting with the PCA reductions showed that windowing the images around $\alpha$ Cen A to a $5^{\prime \prime} \times 5^{\prime \prime}$ enhanced the performance of the PSF subtraction. Indeed, given that the region of interest does not include the region where the center of $\alpha$ Cen B falls, excluding this region avoids the bias that $\alpha$ Cen $\mathrm{B}$ induces in the reference PSF computation with PCA.

Although nominal values for readout noise, photon noise from the sky and telescope background (Ressler et al. 2008; Boccaletti et al. 2015; Rieke et al. 2015) were added to the

\footnotetext{
22 The ETC shows that the wings of the unattenuated $\alpha$ Cen B are not saturated beyond $1^{\prime \prime}-2^{\prime \prime}$ in $10 \mathrm{~s}$.
} 


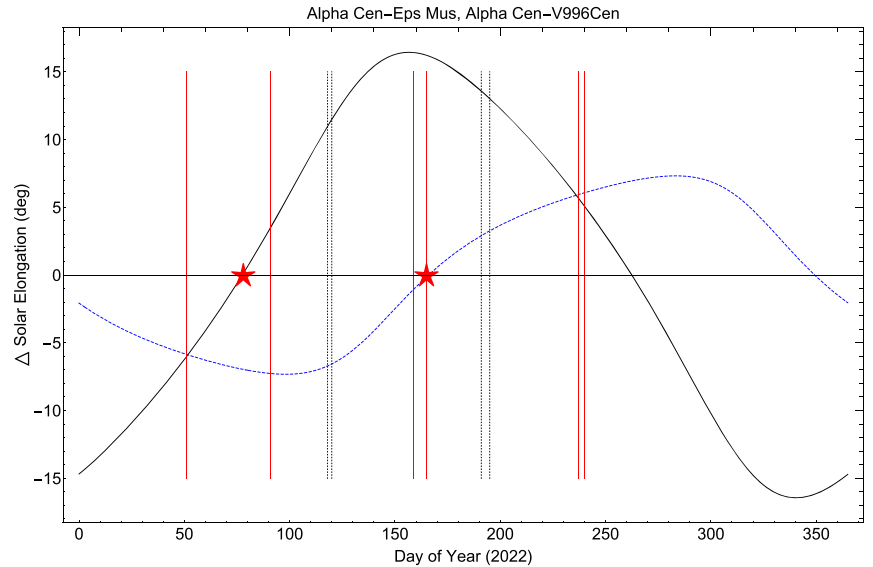

Figure 11. The curves show the difference between the solar elongation angles between $\alpha$ Cen A and two possible reference stars (Table 2), $\epsilon$ Mus (solid black line) and V996 Cen (dotted blue line), through the course of one year, nominally 2022. Pairs of vertical red bars show times when $\alpha$ Cen B can be located within a 4QPM quadrant while the pairs of dotted black bars show times when $\alpha$ Cen B can be hidden behind one of the 4QPM gaps. Minimizing the change in solar elongation angle during a slew between $\alpha$ Cen A and either star is possible on select days marked by red stars. The periods where $\alpha$ Cen B can be placed behind the gap result in slews with large changes in solar elongation angle, $5^{\circ}-10^{\circ}$, between the target and reference stars.

(A color version of this figure is available in the online journal.)

images, the signal from the planet itself and/or speckle noise from $\alpha$ Cen A dominate the measurement within $\sim 3^{\prime \prime}$. The final image had a total integration time of $3.5 \mathrm{hr}$ and was obtained by combining the short exposure frames for $\alpha$ Cen A, $\alpha$ Cen B and one of the simulated planets.

\subsubsection{Minimizing the Effects of Wavefront Drift}

The ability to detect faint companions is dominated by the stability of the nominal $132 \mathrm{~nm}$ of wavefront error (WFE) of the JWST telescope. According to Perrin et al. (2018), a slow-varying thermal WFE ranging from 2 to $10 \mathrm{~nm}$ can be expected depending on the change in solar elongation (and thus in the telescope's thermal balance). Assuming a minimal solar elongation difference as illustrated in Figure 11, we adopted a slow-varying thermal WFE of $2 \mathrm{~nm}$ rms over the total observation of either Cen A or the reference star. The wavefront changes were distributed across small-, medium-, and large spatial scales following the prescription of Lightsey et al. (2018), Perrin et al. (2018). For a scenario requiring a large change of solar elongation angle $\geqslant 10^{\circ}$, we used initial WFE maps for the reference and target stars which differed from one another by a random $2-10 \mathrm{~nm}$.

We simulated two different scenarios of wavefront evolution (Figure 11). In one case $\alpha$ Cen B was located behind one of the 4QPM gaps while in the other $\alpha$ Cen B was located at 45 degrees relative to the 4QPM boundaries. Those two scenarios have different implications for the observations. Putting $\alpha$ Cen B on one of the gaps attenuates the star (Boccaletti et al. 2015;
Danielski et al. 2018) with a positive effect on the level of speckles and photon noise on the final image. However, this option requires a stricter time constraint that limits our ability to optimize the solar elongation difference between the target and its reference star. Thermal models of telescope performance show that large changes in elongation angle produce sudden WFE drifts. These sudden WFEs lead to a higher level of residual speckles, which proves to be very detrimental to sensitivity. Positioning $\alpha$ Cen $\mathrm{B}$ in between two quadrant boundaries relaxes this time constraint and enables us to optimize the difference in solar elongation. In the first scenario with $\alpha$ Cen $\mathrm{B}$ on one of the gaps, the difference in solar elongation is estimated to $\sim 10^{\circ}$, which could result in a wavefront offset between 2 and $10 \mathrm{~nm}$ rms between target and reference star WFE distributions (Perrin et al. 2018) whereas with $\alpha$ Cen $\mathrm{B}$ falling between two quadrant boundaries, the difference in solar elongation can be reduced to near zero which (Perrin et al. 2018) suggests would result in a slowly evolving wavefront difference of $2 \mathrm{~nm}$ rms or less.

Figure 12 compares the $\mathrm{S} / \mathrm{N}$ in the PCA-processed images for different planet radii and temperatures (separations) for wavefront errors of $2 \mathrm{~nm} \mathrm{rms}$ (left). The noise at each radial offset was determined by taking the median of the values within an $1 \lambda / D$ annulus at that radius. The $\mathrm{S} / \mathrm{N}$ drops for smaller planet radius and with increasing star-planet separation due to the decrease in planet temperature. The effective limit $(\mathrm{S} / \mathrm{N} \sim 5)$ of these observations is roughly 5-6 $R_{\oplus}$ within 1 !" 5 . The $10 \mathrm{~nm}$ case (not shown) is even less favorable, strongly favoring observing scenarios which minimize WFE drifts. Figure 12(b) shows a final F1550C image showing both $\alpha$ Cen $\mathrm{B}$ and an inset showing the PCA-corrected region with a $10 R_{\oplus}$ planet at 1.5 from $\alpha$ Cen A.

Our simulations show that the scenario where $\alpha$ Cen B falls on one of the gaps, the change in wavefront stability resulting from large changes solar angle greatly offsets the advantage of lower $\alpha$ Cen B intensity. The scenario where $\alpha$ Cen B falls within a quadrant is more favorable to the detection of small planets.

These results reinforce the fact that, in the present case of direct imaging of exoplanets around $\alpha$ Cen, but also for more general cases for direct imaging of circumstellar environments, optimizing the wavefront stability through the adequate choice of reference star and optimization of observing times is crucial. On a separate note, observing sources off the gap allows observations with the (Angular Differential, ADI) strategy via rolls during a given visit or via multiple visits.

Figure 6 shows that in the present era, when the separation between the two stars is $\sim 7^{\prime \prime}$, the presence of $\alpha$ Cen B has a relatively small effect on the ability to detect a planet orbiting $\alpha$ Cen A. Not until 1" 5 does $\alpha$ Cen B appear to have a significant effect on post-processed contrast ratio, increasing from $5 \times 10^{-5}$ to $8 \times 10^{-5}$ on the $\alpha$ Cen $\mathrm{B}$ facing side.

Finally, we assessed the effect of increasing the integration time within a single visit by a factor of 2 or more and did not 

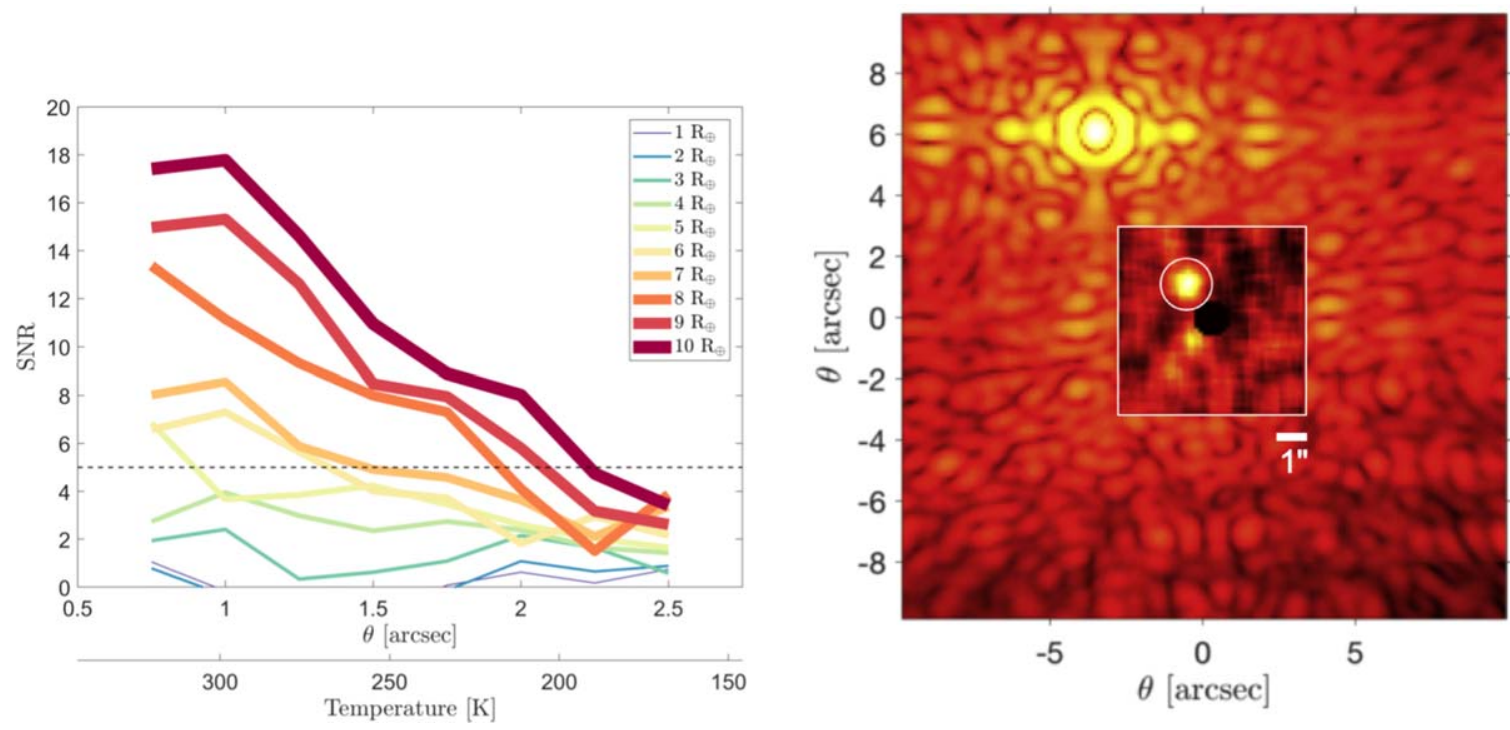

Figure 12. ((a), left) The sensitivities for different planet sizes at the expected angular separation range of detection were computed for a slow thermal varying wavefront error of $2 \mathrm{~nm} \mathrm{rms} \mathrm{(left).} \mathrm{((b),} \mathrm{right)} \mathrm{Simulation} \mathrm{for} \mathrm{the} 2 \mathrm{~nm}$ case (left) for the $\alpha$ Cen system with the F1550C filter centered on $\alpha$ Cen A, with $\alpha$ Cen B on the top left, $7^{\prime \prime}$ away. The PCA reduction of the data is done on a $5^{\prime \prime} \times 5^{\prime \prime}$ central portion of the full image (white square, the scales inside the square are different from outside). A $10 R_{\oplus}$ planet is detected at 1 !" 5 (white circle).

(A color version of this figure is available in the online journal.)

see any improvement in the detectability of smaller planets. Our analysis suggests that the noise floor is set by residual speckle noise, not photon noise. Furthermore, within a given visit, the range of roll angles is modest, $\pm 5^{\circ}$, so that the power of ADI is limited. The maximum $10^{\circ}$ roll results in only a two pixel shift at $1 . \prime 5$, compared with the $0 . \prime 6$ resolution at $15.5 \mu \mathrm{m}$. However, combining multiple visits with a broader range of angles and independent samples of the WFE map and drift, should produce improved sensitivity to small planets. Such visits will be necessary in any event to ensure that any planets obscured within the IWA are observed. Repeating this basic $3.5 \mathrm{hr}$ observing block described here 9 times with independent wavefront realizations could result in a three-fold improvement in sensitivity and allow detections of planets down to $\sim 3 R_{\oplus}$.

\section{Detecting and Imaging the Exozodiacal Cloud}

Observations of the ZodiPic model (Section 4, Figure 5) have been simulated using the observing scenario described above and were reduced using PCA analysis with the results shown in Figure 13. The figure shows the result of a $10 \mathrm{hr}$ exposure. The resolved exozodiacal cloud is readily detectable at levels above $\sim 3$ Zodi (or $\sim 5$ in a single $3.5 \mathrm{hr}$ exposure) and the excess integrated around the entire $\mathrm{HZ}$ would probably be detectable below that level. Detection of a $\mathrm{HZ}$ exozodiacal dust cloud at this level would be a unique contribution by JWST to our knowledge of the environment of the $\mathrm{HZ}$ of a solar type star.

\section{Probability of Detecting a Planet Around $\alpha$ Cen A}

We use a Monte Carlo analysis (Beichman et al. 2010) to assess the probability of finding a planet of a given radius, $R_{p}$ $\left(R_{\oplus}\right)$, and semimajor axis, SMA (au), in the F1550C filter. The flux density of the planet is calculated from the blackbody function (Equation (3)) at the appropriate planet radius and orbital location, $d$. Figure 4 shows the range of planet brightness which approaches a few mJy for $10 R_{\oplus}$ planets. For simplicity we have assumed complete redistribution of absorbed stellar energy so that there is no day-night temperature gradient and no difference in temperature as a function of phase angle. Figure 3 shows that a simple blackbody (Figure 4) over-estimates the brightness of Earth analogs with a deep $\mathrm{CO}_{2}$ absorption feature at $15 \mu \mathrm{m}$. Such planets are already far below the JWST detection limit considered here, so the absorption figure was ignored in the Monte Carlo calculation.

An input population is randomly drawn from the sample described by Equation (1) (Section 2) with the additional constraint of a RV cut of $5 \mathrm{~m} \mathrm{~s}^{-1}$ appropriate to a $100 M_{\oplus}$ planet at 2 au (Zhao et al. 2017). Orbital eccentricity is randomly drawn between $0<$ eccentricity $<0.5$. To convert from planet mass to the planet radius needed to estimate thermal emission, we follow Wolfgang et al. (2016) and adopt $M=C\left(R / R_{\oplus}\right)^{\gamma}$ with values for $C$ and $\gamma$ from their Table 1: $C=1.6 M_{\oplus}$ and $\gamma=1.8$. Similarly, we take the dispersion around the predicted radius is taken from their Equation (3), $\sigma=\sqrt{\sigma_{1}^{2}+\beta\left(R / R_{\oplus}-1\right)}$ with $\sigma_{1}=2.9 M_{\oplus}$ and $\beta=1.5$. 


\section{Contrast to peak}
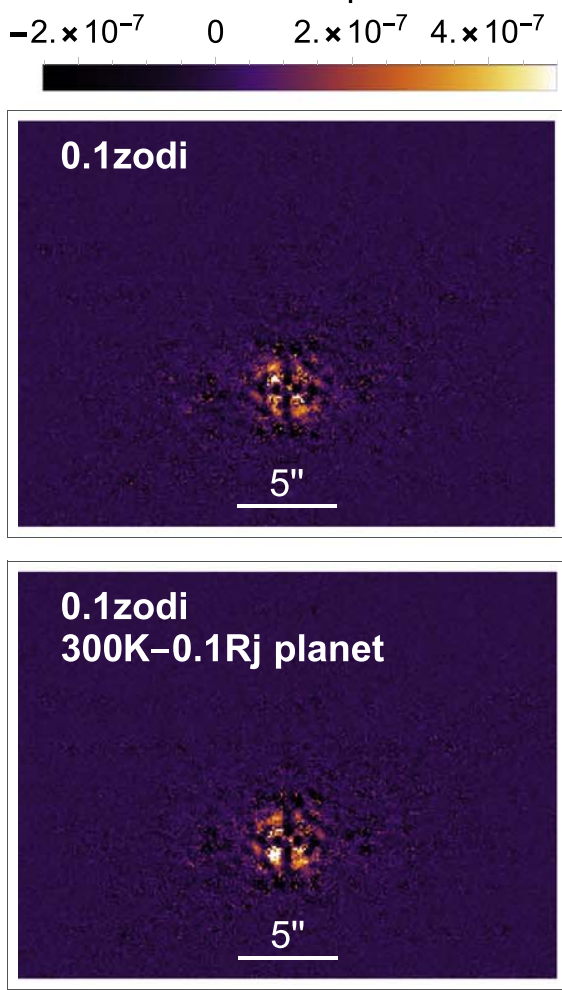

\section{Contrast to peak}
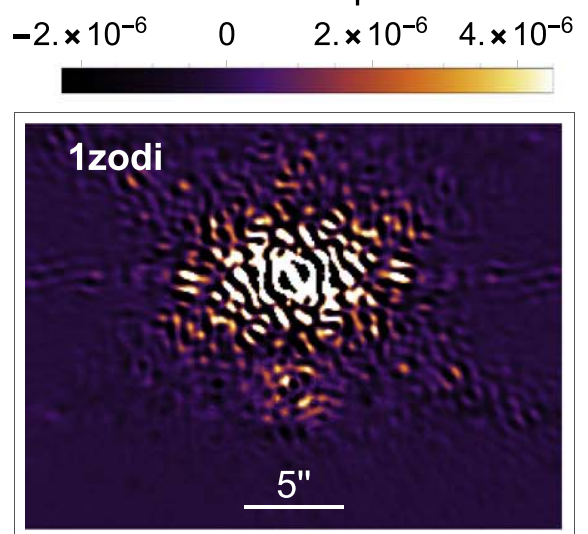

\section{Contrast to peak}
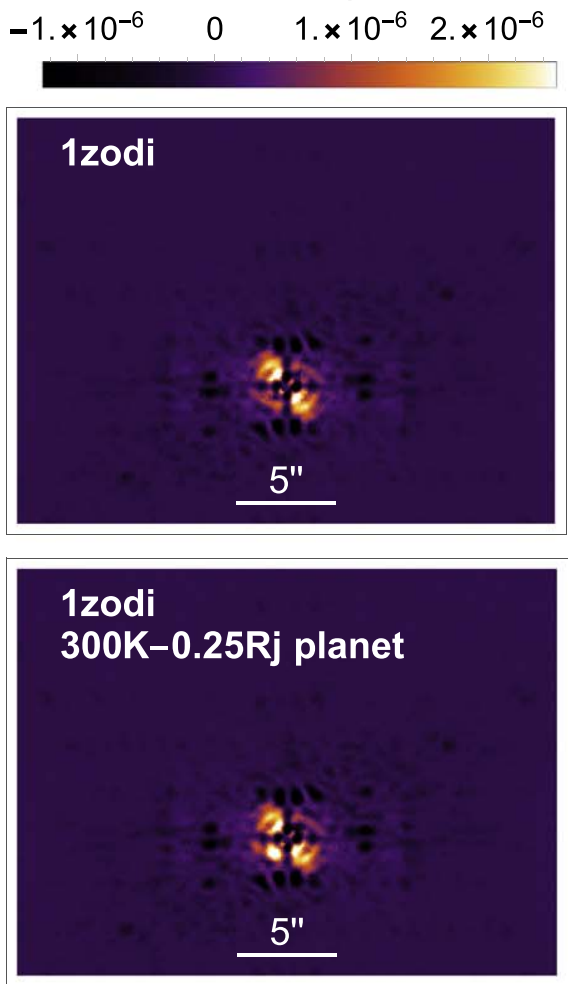

\section{Contrast to peak}
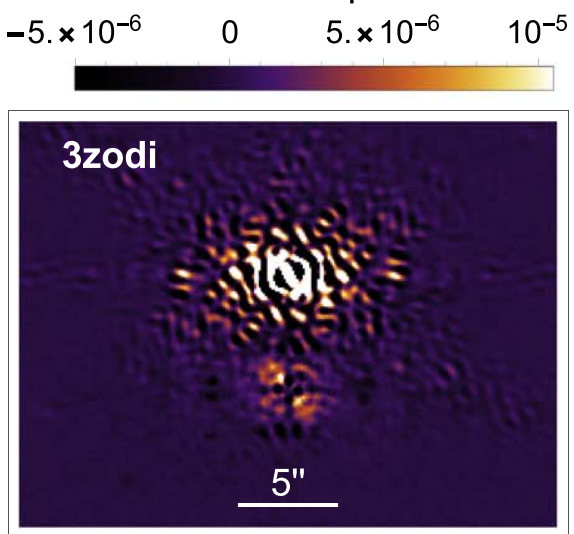

\section{Contrast to peak}

$\begin{array}{llll}-0.00001 & 0 & 0.00001 & 0.00002\end{array}$

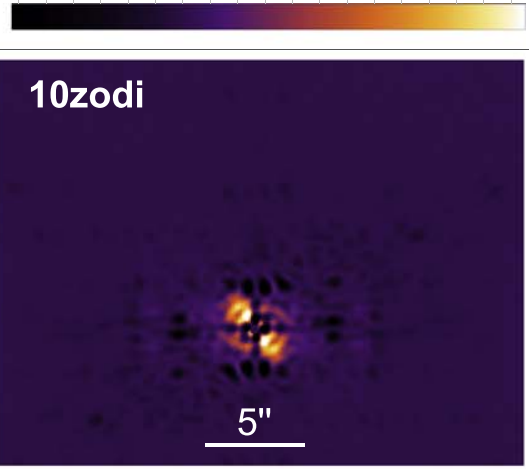

\section{0zodi \\ 300K-1Rj planet}

\section{Contrast to peak}

\section{$\begin{array}{llll}-0.00001 & 0 & 0.00001 & 0.00002\end{array}$}

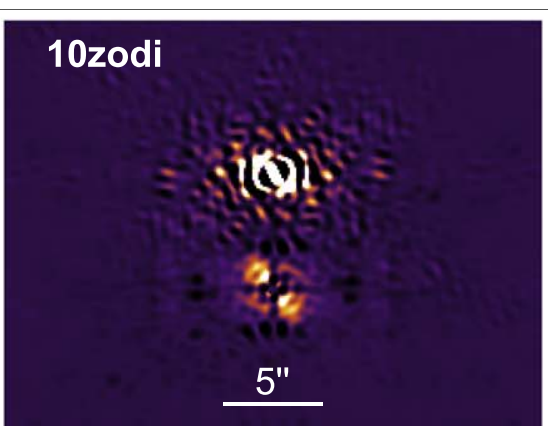

Figure 13. The zodi model (Figure 5) as observed with MIRI using the 4QPM mask in a $10 \mathrm{hr}$ exposure. The top two rows show PCA reductions of observations ignoring the influence of $\alpha$ Cen B. The data were taken using Small Grid Dithers for models with three different levels of zodiacal emission (0.1, 1, 10) Zodi, without and with a $300 \mathrm{~K}$ planet of three different radii $(0.1,0.25$, and 1$) R_{\text {Jup }}$. The bottom panel adds in the effect of $\alpha$ Cen B for the no planet case.

(A color version of this figure is available in the online journal.)

In the simulation planets are placed at randomized locations in their orbits. Planets with apoastron greater than 3 au are excluded due to stability arguments. An apoapse of 3 au is used as a hard limit, because there appear to be no islands of stability beyond that distance (Figure 2, Quarles \& Lissauer 2016). The planets are confined to the plane of the $\alpha$ Cen AB binary system
(Kervella et al. 2016) with an added dispersion in the inclination of $5^{\circ}$. Each planet is started on its orbit at a random time of periastron passage so that the Monte Carlo analysis samples all possible positions of planets relative to the IWA of the MIRI coronagraph. This analysis adopts the transmission of the 4QPM mask (Boccaletti et al. 2015) and the one-dimensional 

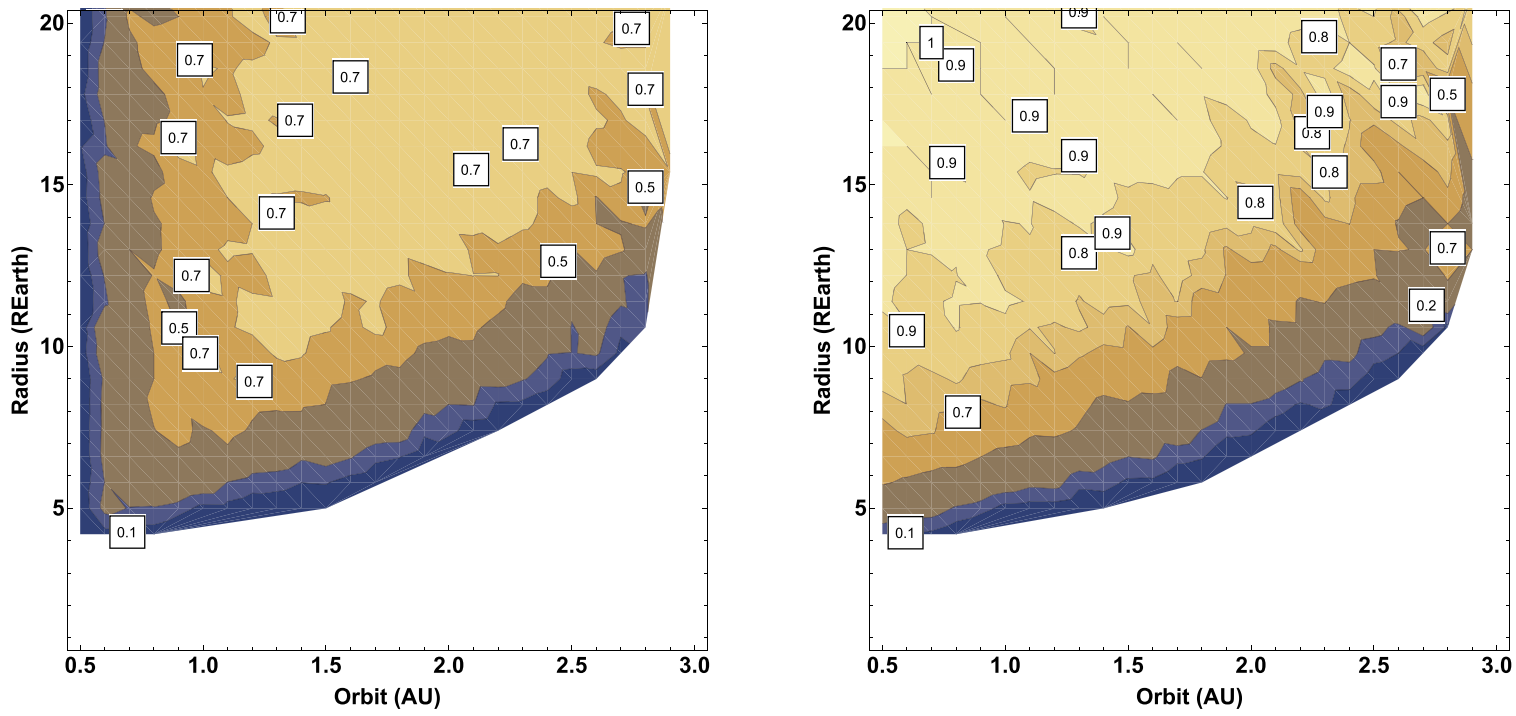

Figure 14. ((a), left) A plot showing the detectablity of planets with a specified radius and semimajor axis (SMA) in a single visit, averaged over ranges of albedo, orbital eccentricity and orientation as described in the text. The contour levels show the fraction of planets detected in a given (Radius, SMA) bin. ((b), right) same plot but showing sensitivity-limited detectability which ignores geometrical incompleteness due to a planet being hidden within the Inner working Angle.

(A color version of this figure is available in the online journal.)

coronagraph performance curve shown in Figure 6 which is based on the PCA post-processing (Section 6.2).

Figure 14(a) shows contours of the probability of detecting a planet of a given radius and semimajor axis in a single visit with $3.5 \mathrm{hr}$ of on-target integration time. There is a broad plateau of detectability $\sim 50 \%$ for $R>5 R_{\oplus}$ and $1<\mathrm{SMA}<2$ au. Figure 14(b) shows detectability contours based purely on photometric considerations, i.e., ignoring geometrical constraint due to planets being obscured within the IWA, and show what planets might be detected in the limit of multiple visits.

Figure 14 does not take into account the restriction on planets due to the RV observations. Figure 15(a) shows a smoothed histogram of all detected planets, similar to Figure 14(b), while Figure 15(b) shows the distribution of planets which could be detected and still be consistent with the $\sim 5 \mathrm{~m} \mathrm{~s}^{-1}$ PRV upper limit. Using the Fernandes et al. (2019) occurrence rates, Equation (1), the fraction of all planets detectable within the $5 \mathrm{~m} \mathrm{~s}^{-1} \mathrm{RV}$ limit and a $5 R_{\oplus}$ MIRI limit is only $5 \%$. A more extensive campaign of multiple visits (with independent wavefront realizations) could push to lower radii and higher completeness (Section 8.1). A $3 R_{\oplus}$ MIRI limit could detect $\sim 13 \%$ of all of the planets expected on the basis of the (poorly) known planet population and consistent with the RV limit; however, as noted in Section 2, the occurrence rates (Fernandes et al. 2019) could be a factor of 3 lower in a binary system (Kraus et al. 2016).

\subsection{Sources of Incompleteness}

Because $\alpha$ Cen $\mathrm{A}$ is seen close to edge on, a planet can be missed because its semimajor axis (or apoastron for an eccentric orbit) never takes it outside the Inner Working Angle of the coronagraph or simply not far enough to be in a region of reduced speckle noise. Thus, the IWA and the contrast limit close to the IWA limit the semimajor axis at which planets can be detected. Second, planets with orbits larger than the IWA can still be missed as they pass behind the IWA in their orbit. Thus, the maximum fractional detectability for a planet at SMA $=1.2$ au $(0, " 9)$ with respect to the IWA of 0 " 49 at $\mathrm{F} 1550 \mathrm{C}$ is $1-\frac{2}{\pi} \operatorname{ArcSin}\left(\frac{\mathrm{IWA}}{\mathrm{SMA}}\right)=63 \%$ in a single visit. As planets move further out, the fraction of time they are missed for geometrical reasons decreases. But, as they move further out, their temperature drops so they might be missed for reasons of low $\mathrm{S} / \mathrm{N}$. These two effects account for the general shape of the detectability in Figure 14(a). The solution to the problems of geometrical incompleteness is carrying out multiple observations over a number of epochs as pointed out in many studies of this question (Brown 2005, 2015).

Two additional sources of incompleteness are not accounted for in Figure 14. First is the increased noise level in the direction of $\alpha$ Cen B and second from the possibility that at any one instant a planet may hide behind one of the 4QPM's quadrant gaps. Figure 6 shows remarkably little difference in the post-processing curves in the direction of $\alpha$ Cen $\mathrm{B}$ relative to other directions within the region of interest, $<3$ au. $\alpha$ Cen $\mathrm{A}$ is simply overpowering at these separations relative to $\alpha$ Cen $\mathrm{B}$ located $7 "-8$ " away.

The second source of incompleteness not taken into account in Figure 14 are the dead areas defined by the 4QPM gaps. We test the second source by performing numerical simulations 

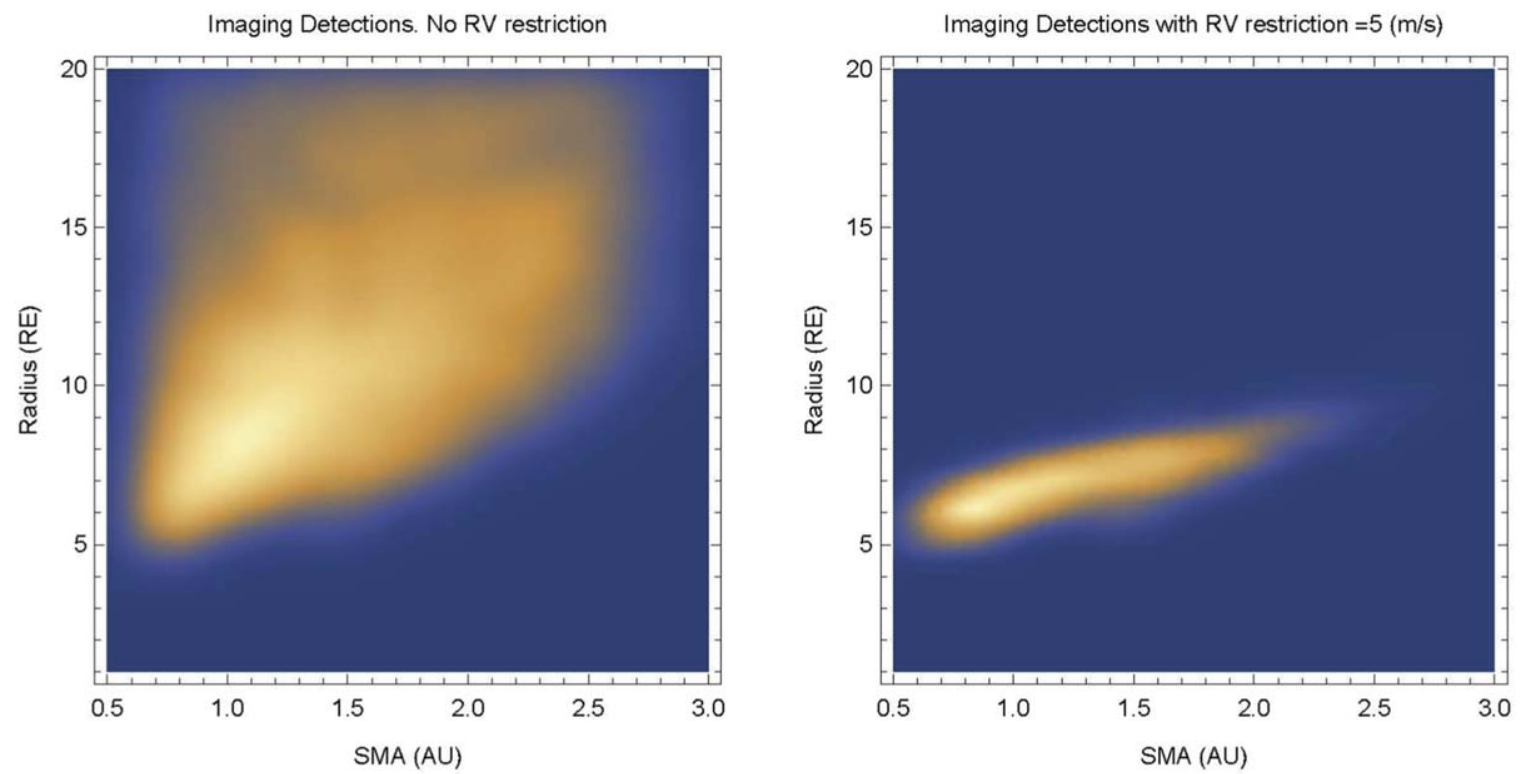

Figure 15. ((a), left) The locus of all potentially detectable planets in (Radius-SMA) space similar to Figure 14(b). ((b), right) The locus of all detected planets subject to the RV limit of $5 \mathrm{~m} \mathrm{~s}^{-1}$. The intensity scale is arbitrary.

(A color version of this figure is available in the online journal.)

using a modified version of the mercury6 integration package designed to evolve planetary orbits in binary systems (Chambers et al. 2002). These simulations use the orbital solution from Pourbaix \& Boffin (2016) for the binary orbit and evaluate the stability on $10^{5} \mathrm{yr}$ timescale for Earth-mass planets over a range of initial SMAs (1-3 au), eccentricity vectors $\left(e_{p} \cos \omega_{p} \leqslant 0.9, \quad e_{p} \cos \omega_{p} \leqslant 0.9\right)$, and mutual inclinations $\left(<90^{\circ}\right)$. Figure 16 shows the projection of initial conditions that are stable (survive for $10^{5} \mathrm{yr}$ ) and binned using the expected angular resolution $(\sim 0$ "! 3$)$ at $15.5 \mu \mathrm{m}$ to identify a normalized number density of potential orbits on the sky plane (see color scale). Projecting the gap width onto the $\sim 2.5$ au zone of stability (Quarles \& Lissauer 2016) reveals that the incompleteness due to the gaps outside of the IWA is around $24 \%$. This source of incompleteness can be mitigated by multiple visits at different orientations. Aligning the gaps with the $\alpha$ Cen $\mathrm{AB}$ axis results in an incompleteness of $60 \%-$ another reason to avoid this observing scenario.

\subsection{Comparison with Ground-based Initiatives}

The proximity of the $\alpha$ Cen system makes it a compelling target for ground-based studies in the $N(10 \mu \mathrm{m})$ band despite the high sky background. As described in Kasper et al. (2017) and Käufl et al. (2018), the European Southern Observatory (ESO) in collaboration with the Breakthrough Initiative has modified the VLT mid-IR imager VISIR to enhance its ability

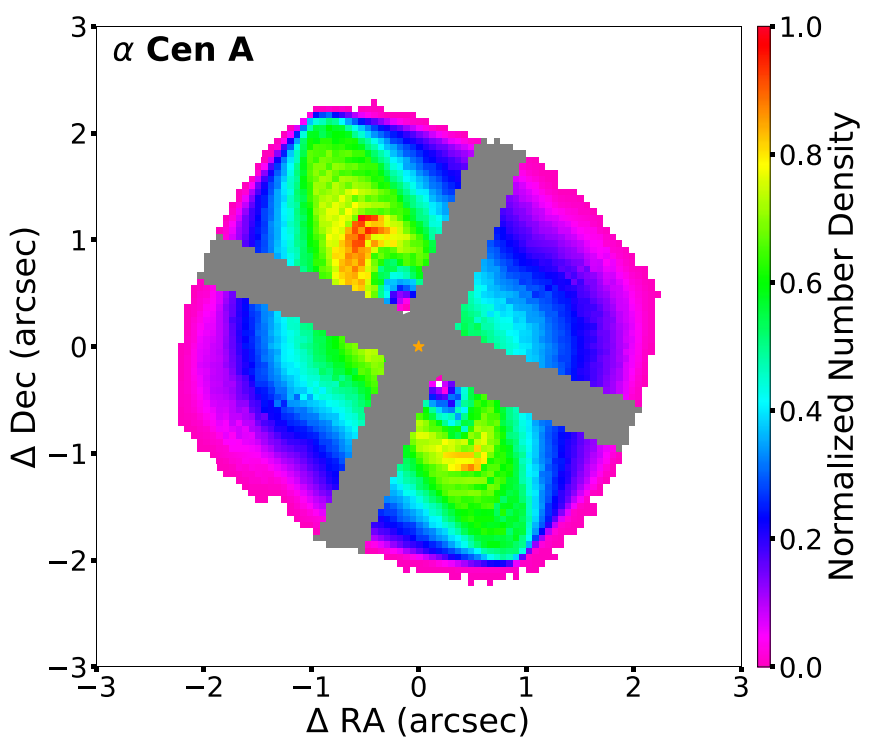

Figure 16. Projection of initial conditions that are stable on $10^{5} \mathrm{yr}$ timescales onto the sky plane. The stable initial conditions are binned for resolution at $15.5 \mu \mathrm{m}$, where the color scale denotes a normalized number density of stable initial conditions within each bin. Bins that do not contain any stable initial conditions are colored white. The regions defined by the 4QPM mask and the gaps between adjacent quadrants are plotted in gray over the region of potential planet stability. These regions block roughly $24 \%$ of the coronagraphic field outside of the IWA.

(A color version of this figure is available in the online journal.) 
to search for potentially habitable planets around both components of $\alpha$ Cen. The NEAR (New Earths in the Alpha Cen Region) concept combines adaptive optics using the deformable secondary mirror at UT4, a new vector vortex coronagraph (Mawet et al. 2005) optimized for the most sensitive spectral bandpass in the $N$-band, and fast chopping for noise filtering.

The recently demonstrated sensitivity of the NEAR instrument is $650 \mu \mathrm{Jy}$ ( $5 \sigma$ in $1 \mathrm{hr}$, Käufl et al. 2018). Assuming no systematic errors intervene, a $100 \mathrm{hr}$ observing program with NEAR could have the sensitivity to detect a $2 R_{\oplus}$ planet with an Earth-like emission spectrum at $\sim 3 \lambda / D \sim 1$ au and a temperature around $300 \mathrm{~K}$. This result, if achieved, could complement $J W S T$ 's MIRI search by extending inward to smaller, hotter planets. In the long term, the NEAR experiment is relevant for the Extremely Large Telescope/METIS instrument (Quanz et al. 2015) which would benefit from the telescope diameter $(D), D^{1}$ gain in inner working angle and the $D^{4}$ gain in photometric sensitivity due to the ELT's $39 \mathrm{~m}$ aperture.

Dynamical searches for planets orbiting $\alpha$ Cen $\mathrm{A}$ are continuing. The new generation of PRV instruments such as ESPRESSO (González Hernández et al. 2018) should be able measure down to a few Earth masses, although the presence of $\alpha$ Cen B presents observational challenges at binary separations smaller than a few arcseconds. On the other hand, both the ALMA and the VLT Gravity interferometers are taking advantage of this binarity by searching for a planet-induced astrometric wobble in the separation between $\alpha$ Cen A and B at millimeter (Akeson et al. 2019) and near-IR wavelengths (Gravity Collaboration et al. 2017), respectively. Dynamical detections from any of these techniques would add critical information on the mass and orbit of any planet found via direct imagedwhether from JWST or other experiments now underway.

\section{Conclusions}

With careful observation planning and advanced postprocessing techniques JWST's MIRI coronagraph could detect planets as small as $5 R_{\oplus}$ at $15.5 \mu \mathrm{m}$ in a single $\sim 20 \mathrm{hr}$ visit (combining $\sim 3.5 \mathrm{hr}$ of on-target integration plus reference star and other overheads). Multiple visits would enhance completeness, provide astrometric confirmation, and push to still lower planet radii. These additional observations would also help to refine orbital data and open a search for additional planets. Detection at MIRI wavelengths would lead to an estimate of the planet's effective temperature and thus its radius which would depend only weakly on the assumed albedo. Of course, the actual performance of JWST in terms of wavefront error and especially WFE stability remains unknown as does the performance of its detectors. A more sustained campaign could push this radius limit down to $\sim 3 R_{\oplus}$.

MIRI could also detect an exozodiacal dust cloud at the level of $3 \sim 5 \times$ the brightness of our own cloud. Depending on the strength and distribution of the exozodiacal dust, such emission could mask the light of any planet.

JWST data, in conjunction with ground-based observations would provide refined characterization of any detected planets: PRV measurements with both current and next generation instruments such as CHIRON and ESPRESSO (Zhao et al. 2017; González Hernández et al. 2018) would yield a refined orbit and the planet's mass from which we would determine its bulk composition; VLT/NEAR detections at shorter wavelengths, $\sim 10 \mu \mathrm{m}$, would refine the spectral energy distribution. Ultimately, instruments combining high contrast imaging with high spectral resolution spectroscopy on $30-40 \mathrm{~m}$ telescopes would open up the prospect of exoplanet spectroscopy of a planet orbiting in the HZ of a solar type star (Snellen et al. 2015; Wang et al. 2017).

Some of the research described in this publication was carried out in part at the Jet Propulsion Laboratory, California Institute of Technology, under a contract with the National Aeronautics and Space Administration. Copyright 2019 California Inst of Technology. All rights reserved.

\section{Appendix MIRI Detector Tests}

Estimates based on the JWST Exposure Time Calculator $\left(\mathrm{ETC}^{23}\right)$ for the unattenuated signal from $\alpha$ Cen B is approximately $5 \times 10^{7}$ electrons s$^{-1}$ at $15.5 \mu \mathrm{m}$ which is well above the saturation limit for the MIRI detectors. To explore the implications of such a bright source in the focal plane we carried out a series of tests using the flight-like configuration at JPL. Figure 17 shows the resulting image from the bright target test. The test setup was set so that it would quickly saturate the detector with a signal of a factor of 10 more than the saturation limit. Figure 17 plots the signal recorded for one illuminated pixel for each of the 30 groups in one integration. The test source saturated the detector in 4 groups or approximately $11 \mathrm{~s}$ for a total time per integration of $80 \mathrm{~s}$. The total exposure time of 10 integrations was 14 minutes.

The resulting test image (Figure 17) shows a good detection of the 3 sources used in the test despite the "super" saturation of the detector. Other than glints and optical effects which originate in the test bench setup, there is no significant impact in the image quality from the "super-saturation" of the detector. In Figure 18 we set the scale of the test image to enhance the background to reveal faint structures associated with the rows and columns of the detected sources. Row profiles of the image, also presented in Figure 18, show that the background artifacts are 4 orders of magnitude lower in flux than the sources in the image.

This row and column structure in the detectors had been previously identified by the MIRI test team and is associated

\footnotetext{
${ }^{23}$ https://jwst.etc.stsci.edu/
} 

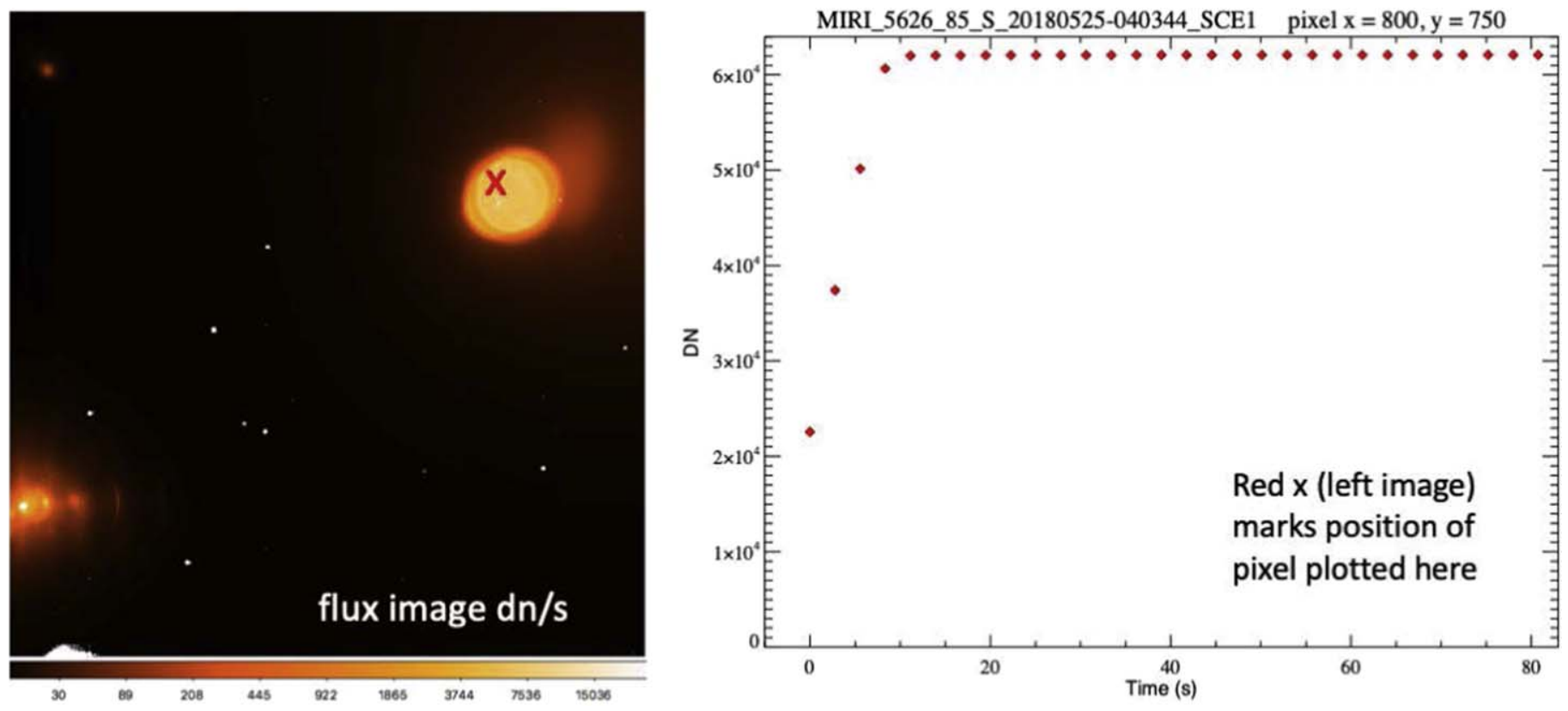

Figure 17. (Left) Full frame MIRI engineering model detector. The tests includes two sources from a masked blackbody, a faint point like object in the top left, and an extended disk structure in the top right. An unfocussed LED source can be seen in the bottom left of the image. The units of the image are flux (Data Numbers, DN/s) as calculated from the slope of unsaturated frames. (Right) The signal recorded from one pixel in the disk blackbody source.

(A color version of this figure is available in the online journal.)
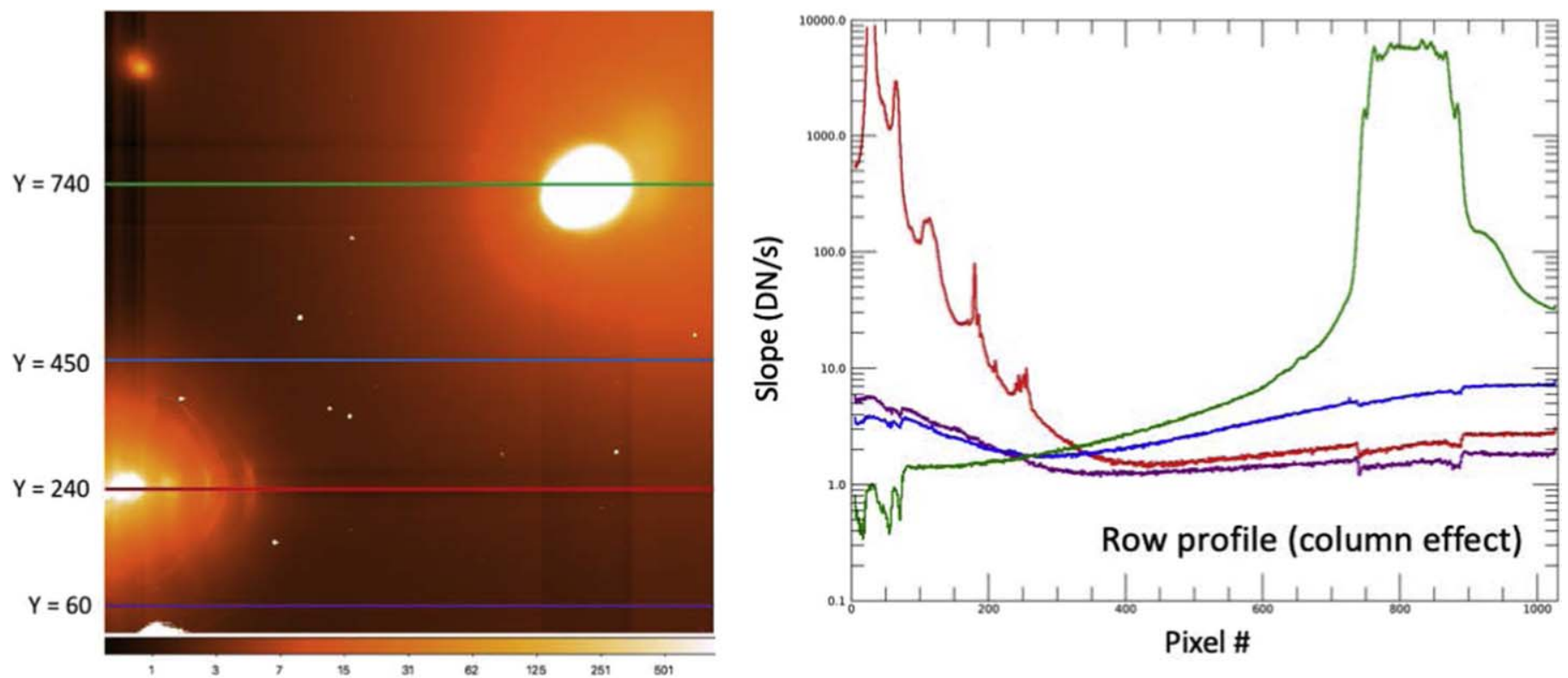

Figure 18. ((a), left) The same test data as Figure 17 but with the scale set to highlight faint structure in the background. (b) Colored lines crossing the image horizontally mark rows whose intensities are shown on the right. ((b), right) The profiles of the marked rows in the detector showing the effects of the column effect in the rows underneath of brightest source.

(A color version of this figure is available in the online journal.) 

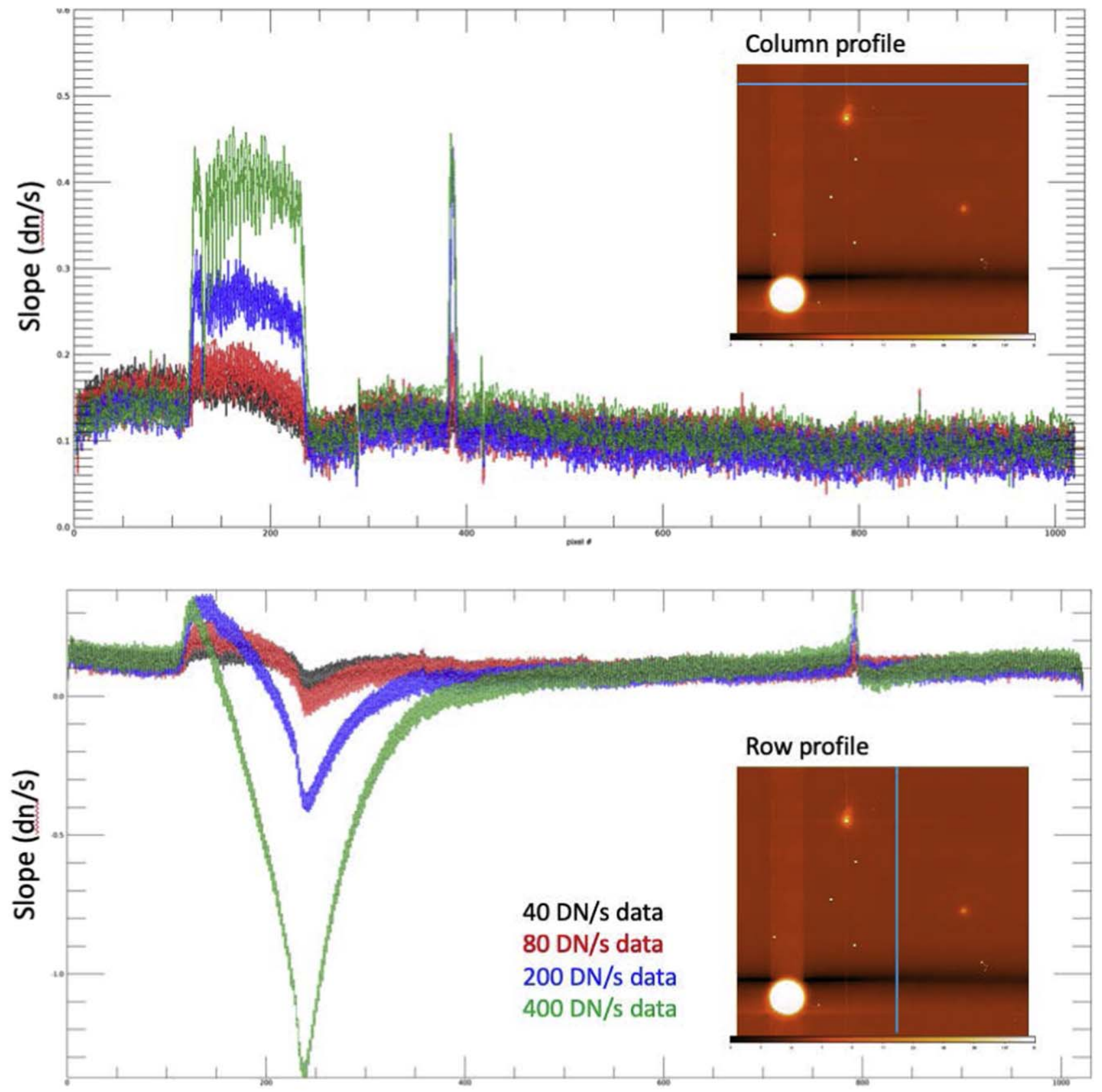

Pixel \#

Figure 19. JPL MIRI test data results showing data with 3 blackbody sources at four different flux levels. Row (bottom) and column (top) profiles at each flux level highlight the extent of the artifacts in each direction.

(A color version of this figure is available in the online journal.)

with bright source detections (Figure 19). We believe the artifacts will have little impact on the detection of planets the following reasons. First, the row and column artifacts are accentuated in the JPL test images due to the very low backgrounds in the test conditions- $\mathrm{a}$ factor of three less than we expect at the shortest MIRI wavelength range of $5.7 \mu \mathrm{m}$. For the higher backgrounds expected from the MIRI 4QPMs (F1550C) the column and row effects will be significantly diluted. (Figure 19) also shows that, although the effect is flux dependent, it is limited to the columns in which there are bright 
sources, therefore the effect from $\alpha$ Cen B should be limited to the columns in which it is placed.

However, the row effect extends beyond the source rows, in the read direction up the detector, with a dependence on source size. Therefore, there is a possibility that the row artifact could affect planet detection if $\alpha$ Cen B were placed in a lower quadrant of the 4QPM. However, we expect the point like nature of $\alpha$ Cen B will help reduce the amplitude of this artifact. Lastly, the artifacts have shown to be highly uniform in amplitude in the row and column direction, therefore preliminary efforts to correct the image based on median column and row filtering have proved promising. In summary, we find no limitations from the point of view of MIRI detectors to the detection of planets around the $\alpha$ Cen $\mathrm{AB}$ system from MIRI ground detector testing. Including the case of super saturation, which is expected in the observation of the $\alpha$ Cen AB system with the MIRI 4QPMs.

\section{ORCID iDs}

Charles Beichman (10 https://orcid.org/0000-0002-5627-5471

Marie Ygouf (i) https://orcid.org/0000-0001-7591-2731

Dimitri Mawet (1) https://orcid.org/0000-0002-8895-4735

Yuk Yung (i) https://orcid.org/0000-0002-4263-2562

Elodie Choquet $\odot$ https://orcid.org/0000-0002-9173-0740

Pierre Kervella (1) https://orcid.org/0000-0003-0626-1749

Anthony Boccaletti (i) https://orcid.org/0000-0001-9353-2724

Jack J. Lissauer (1) https://orcid.org/0000-0001-6513-1659

Renyu Hu (1) https://orcid.org/0000-0003-2215-8485

Bertrand Mennesson (1) https://orcid.org/0000-0003-4205-4800

Jarron Leisenring (1) https://orcid.org/0000-0002-0834-6140

\section{References}

Akeson, A., Beichman, C. A., Fomalont, E., Kervella, P., \& Benedict, G. F. 2019, in Presented at 2019 Astrobiology Science Conf. (AbSciCon), https://agu. confex.com/agu/abscicon19/meetingapp.cgi/Paper/482402

Amara, A., \& Quanz, S. P. 2012, MNRAS, 427, 948

Angel, J. R. P., \& Woolf, N. J. 1997, ApJ, 475, 373

Beichman, C. A., Bryden, G., Gautier, T. N., et al. 2005, ApJ, 626, 1061

Beichman, C. A., Bryden, G., Stapelfeldt, K. R., et al. 2006a, ApJ, 652, 1674

Beichman, C. A., Fridlund, M., Traub, W. A., et al. 2007, in Protostars and Planets V, ed. B. Reipurth, D. Jewitt, \& K. Keil (Tucson: Univ. Arizona Press), 915

Beichman, C. A., Krist, J., Trauger, J. T., et al. 2010, PASP, 122, 162

Beichman, C. A., Tanner, A., Bryden, G., et al. 2006b, ApJ, 639, 1166

Belikov, R., Bendek, E., Thomas, S., Males, J., \& Lozi, J. 2015, Proc. SPIE, 9605, 960517

Boccaletti, A., Lagage, P.-O., Baudoz, P., et al. 2015, PASP, 127, 633

Brown, R. A. 2005, ApJ, 624, 1010

Brown, R. A. 2015, ApJ, 799, 87

Burrows, A., Sudarsky, D., \& Hubeny, I. 2004, ApJ, 609, 407

Burrows, A. S. 2014, PNAS, 111, 12601

Cahoy, K. L., Marley, M. S., \& Fortney, J. J. 2010, ApJ, 724, 189

Chambers, J. E., Quintana, E. V., Duncan, M. J., \& Lissauer, J. J. 2002, AJ, 123, 2884

Churchwell, E., Babler, B. L., Meade, M. R., et al. 2009, PASP, 121, 213

Cowley, W. I., Baugh, C. M., Cole, S., Frenk, C. S., \& Lacey, C. G. 2018, MNRAS, 474, 2352

Cumming, A., Butler, R. P., Marcy, G. W., et al. 2008, PASP, 120, 531

Danielski, C., Baudino, J.-L., Lagage, P.-O., et al. 2018, AJ, 156, 276
Des Marais, D. J., Harwit, M. O., Jucks, K. W., et al. 2002, AsBio, 2, 153 Ertel, S., Defrère, D., Hinz, P., et al. 2018, AJ, 155, 194

Fazio, G., \& Megeath, T. 2004, sptz prop, 3911680

Fernandes, R. B., Mulders, G. D., Pascucci, I., et al. 2019, ApJ, 874, 81

González Hernández, J. I., Pepe, F., Molaro, P., et al. 2018, Handbook of Exoplanets (Berlin: Springer), 157

Gravity Collaboration, Abuter, R., Accardo, M., et al. 2017, A\&A, 602, A94

Holman, M. J., \& Wiegert, P. A. 1999, AJ, 117, 621

Howard, A. W. 2013, Sci, 340, 572

Hu, R., Ehlmann, B. L., \& Seager, S. 2012, ApJ, 752, 7

Hu, R., Seager, S., \& Bains, W. 2013, ApJ, 769, 6

Hu, R., Seager, S., \& Bains, W. 2014, ApJ, 784, 63

Kasper, M., Arsenault, R., Kaufl, H.-U., et al. 2017, Msngr, 169, 16

Käufl, H.-U., Kasper, M., Arsenault, R., et al. 2018, Proc. SPIE, 10702, 107020D

Kenworthy, M. A., Absil, O., Agócs, T., et al. 2016, Proc. SPIE, 9908, 9908A6 Kervella, P., Bigot, L., Gallenne, A., et al. 2017, A\&A, 597, A137

Kervella, P., Mignard, F., Mérand, A., \& Thévenin, F. 2016, A\&A, 594, A107

Knight, J. S., Lightsey, P., \& Barto, A. 2012, Proc. SPIE, 8442, 84422G

Kopparapu, R. k., Wolf, E. T., Arney, G., et al. 2017, ApJ, 845, 5

Kraus, A. L., Ireland, M. J., Huber, D., Mann, A. W., \& Dupuy, T. J. 2016, AJ, 152,8

Kuchner, M. 2012, ZODIPIC: Zodiacal Cloud Image Synthesis Astrophysics Source Code Library ascl:1202.002

Léger, A., Mariotti, J. M., Mennesson, B., et al. 1996, Icar, 123, 249

Lightsey, P. A., Knight, J. S., Barto, A., et al. 2018, Proc. SPIE, 10698, 1069804

Mawet, D., Hirsch, L., Lee, E. J., et al. 2019, AJ, 157, 33

Mawet, D., Riaud, P., Absil, O., et al. 2005, ApJ, 633, 1191

Mawet, D., Wizinowich, P., Dekany, R., et al. 2016, Proc. SPIE, 9909, 99090D

Mazin, B., Artigau, E., Bailey, V., et al. 2019, BAAS, 51, 128

Mennesson, B., Gaudi, S., Seager, S., et al. 2016, Proc. SPIE, 9904, 99040L

National Academies of Sciences 2018, Exoplanet Science Strategy Report (Washington, DC: The National Academies Press)

National Research Council 2010, New Worlds, New Horizons in Astronomy and Astrophysics (Washington, DC: The National Academies Press)

Olnon, F. M., Raimond, E., Neugebauer, G., et al. 1986, A\&AS, 65, 607

Perrin, M. D., Pueyo, L., Van Gorkom, K., et al. 2018, Proc. SPIE, 10698, 1069809

Pourbaix, D., \& Boffin, H. M. J. 2016, A\&A, 586, A90

Pueyo, L., Zimmerman, N., Bolcar, M., et al. 2017, Proc. SPIE, 10398, 103980F

Quanz, S. P., Crossfield, I., Meyer, M. R., Schmalzl, E., \& Held, J. 2015, IJAsB, 14, 279

Quarles, B., \& Lissauer, J. J. 2016, AJ, 151, 111

Quarles, B., \& Lissauer, J. J. 2018, AJ, 155, 130

Quarles, B., Lissauer, J. J., \& Kaib, N. 2018, AJ, 155, 64

Quintana, E. V., Lissauer, J. J., Chambers, J. E., \& Duncan, M. J. 2002, ApJ, 576, 982

Ressler, M. E., Cho, H., Lee, R. A. M., et al. 2008, Proc. SPIE, 7021, 702100 Ressler, M. E., Sukhatme, K. G., Franklin, B. R., et al. 2015, PASP, 127, 675 Rieke, G. H., et al. 2015, PASP, 127, 665R

Santerne, A., Moutou, C., Tsantaki, M., et al. 2016, A\&A, 587, A64

Seager, S., \& Deming, D. 2010, ARA\&A, 48, 631

Skemer, A., Stelter, D., Mawet, D., et al. 2018, Proc. SPIE, 10702, 10702A5

Snellen, I., de Kok, R., Birkby, J. L., et al. 2015, A\&A, 576, A59

Soummer, R., Pueyo, L., \& Larkin, J. 2012, ApJL, 755, L28

Su, K. Y. L., Rieke, G. H., Malhotra, R., et al. 2013, ApJ, 763, 118

Teplitz, V. L., Stern, S. A., Anderson, J. D., et al. 1999, ApJ, 516, 425

Thévenin, F., Provost, J., Morel, P., et al. 2002, A\&A, 392, L9

Thompson, S. E., Coughlin, J. L., Hoffman, K., et al. 2018, ApJS, 235, 38

Traub, W. A., \& Oppenheimer, B. R. 2010, Exoplanets (Berlin: Springer), 111 Turnbull, M. C. 2015, arXiv:1510.01731

Turnbull, M. C., Traub, W. A., Jucks, K. W., et al. 2006, ApJ, 644, 551

Wang, J., Mawet, D., Ruane, G., et al. 2017, AJ, 153, 183

Wiegert, J., Liseau, R., Thébault, P., et al. 2014, A\&A, 563, A102

Wolfgang, A., Rogers, L. A., \& Ford, E. B. 2016, ApJ, 825, 19

Xie, J.-W., Zhou, J.-L., \& Ge, J. 2010, ApJ, 708, 1566

Zhang, Y., Li, Q., Xie, J.-W., et al. 2018, ApJ, 861, 116

Zhao, L. L., Fischer, D. A., Brewer, J. M., et al. 2017, arXiv:1711.06320 